\title{
Diffractive behavior of the wave equation in periodic media: weak convergence analysis
}

\author{
Grégoire Allaire · Mariapia Palombaro · Jeffrey Rauch
}

Received: 23 November 2007 / Published online: 13 September 2008

(c) Fondazione Annali di Matematica Pura ed Applicata and Springer-Verlag 2008

\begin{abstract}
We study the homogenization and singular perturbation of the wave equation in a periodic media for long times of the order of the inverse of the period. We consider initial data that are Bloch wave packets, i.e., that are the product of a fast oscillating Bloch wave and of a smooth envelope function. We prove that the solution is approximately equal to two waves propagating in opposite directions at a high group velocity with envelope functions which obey a Schrödinger type equation. Our analysis extends the usual WKB approximation by adding a dispersive, or diffractive, effect due to the non uniformity of the group velocity which yields the dispersion tensor of the homogenized Schrödinger equation.
\end{abstract}

Keywords Homogenization · Bloch waves · Diffractive geometric optics

Mathematics Subject Classification (2000) $\quad 35 \mathrm{~B} 27 \cdot 35 \mathrm{~J} 10$

\section{Introduction}

The homogenization of the wave equation in periodic media is a well studied subject (see e.g. $[9,10,17,19,28]$ ). It is known that, for non oscillating initial data (often called low frequency data), the homogenized limit is again a wave equation with effective coefficients that can be computed as in the static case. On the other hand, for oscillating initial data in resonance with

G. Allaire $(\varangle)$

Centre de Mathématiques Appliquées, École Polytechnique, 91128 Palaiseau, France

e-mail: gregoire.allaire@polytechnique.fr

M. Palombaro

Max Planck Institute for Mathematics in the Sciences, Inselstrasse 22-26, 04103 Leipzig, Germany

e-mail: Mariapia.Palombaro@mis.mpg.de

J. Rauch

Department of Mathematics, University of Michigan, Ann Arbor, MI 48109, USA

e-mail: rauch@umich.edu 
the periodic medium (so-called high frequency data), the usual two-scale asymptotic method breaks down and one needs to use the famous WKB method (Wentzel, Kramers, Brillouin) to deduce that the asymptotic limit of the wave equation is described by geometric optics, i.e. eikonal equations for the phases and transport equations for the amplitudes of the waves (see e.g. $[9,17]$ or Sect. 6 below for a brief account).

The present work pertains to the second category, namely homogenization with high frequency data. However, the novelty is that we are interested in a much longer time scale, way beyond the geometric optic regime. In this new limit regime, called diffractive or dispersive regime [14-16], the phase is still the solution of the WKB eikonal equation but the amplitude, or envelope function, is not any longer solution of a transport equation but rather solution of a Schrödinger type equation (in a moving frame of reference). Therefore, our homogenized model describes dispersive properties of the wave equation for very long times (as already recognized in a special case in [2]). More precisely, we study the homogenization of the singularly perturbed wave equation

$$
\begin{cases}\varepsilon^{2} \frac{\partial}{\partial t}\left(\rho_{\varepsilon} \frac{\partial u_{\varepsilon}}{\partial t}\right)-\operatorname{div}\left(A_{\varepsilon} \nabla u_{\varepsilon}\right)=0 & \text { in } \mathbb{R}^{N} \times(0, T), \\ u_{\varepsilon}(0, x)=u_{\varepsilon}^{0}(x) & \text { in } \mathbb{R}^{N}, \\ \frac{\partial u_{\varepsilon}}{\partial t}(0, x)=u_{\varepsilon}^{1}(x) & \text { in } \mathbb{R}^{N},\end{cases}
$$

where $T>0$ is a final time, $A_{\varepsilon}$ and $\rho_{\varepsilon}$ are oscillating coefficients of the form

$$
A_{\varepsilon}(x)=A_{0}\left(\frac{x}{\varepsilon}\right)+\varepsilon^{2} A_{1}\left(t, \frac{t}{\varepsilon}, x, \frac{x}{\varepsilon}\right), \quad \rho_{\varepsilon}(x)=\rho_{0}\left(\frac{x}{\varepsilon}\right)+\varepsilon^{2} \rho_{1}\left(t, \frac{t}{\varepsilon}, x, \frac{x}{\varepsilon}\right),
$$

with $\rho_{0}(y)$ and $A_{0}(y)$, real bounded periodic functions of period $(0,1)^{N}$ such that the density $\rho_{0}$ is strictly positive and the tensor $A_{0}$ is symmetric uniformly coercive (see Sect. 2 for precise assumptions). The macroscopic modulations $\rho_{1}(t, \tau, x, y)$ and $A_{1}(t, \tau, x, y)$ are smooth bounded functions which are periodic of period $(0,1)^{N}$ with respect to $y$ (they also satisfy assumption (1.7) below). The second order time derivative in (1.1) has been written in conservative form because the density $\rho_{\varepsilon}$ may depend on time. Of course, if $\rho_{\varepsilon}$ is independent of time, the inertial term is just equal to $\varepsilon^{2} \rho_{\varepsilon}\left(\partial^{2} u_{\varepsilon}\right) /\left(\partial t^{2}\right)$ as usual. There is also an $\varepsilon^{2}$ scaling factor in front of the time derivative which corresponds to very long time. Indeed, upon introduction of a new time variable $\tau=\varepsilon^{-1} t$, the usual wave equation (without scaling) is recovered. Thus a time $t$ of order 1 is equivalent to a long time $\tau$ of order $\varepsilon^{-1}$ (see Sect. 6).

We consider the following type of high-frequency, with linear phase initial data

$$
u_{\varepsilon}^{0}(x)=\psi_{n}\left(\frac{x}{\varepsilon}, \theta_{0}\right) \mathrm{e}^{2 i \pi \frac{\theta_{0} \cdot x}{\varepsilon}} v_{0}(x) \text { and } u_{\varepsilon}^{1}(x)=\frac{1}{\varepsilon^{2}} \psi_{n}\left(\frac{x}{\varepsilon}, \theta_{0}\right) \mathrm{e}^{2 i \pi \frac{\theta_{0} \cdot x}{\varepsilon}} v_{1}(x),
$$

where $v_{0}$ and $v_{1}$ are sufficiently smooth functions and $\psi_{n}$ is a so-called Bloch eigenfunction, solution of the following spectral cell equation in the unit torus $\mathbb{T}^{N}$

$$
-\left(\operatorname{div}_{y}+2 i \pi \theta\right)\left(A_{0}(y)\left(\nabla_{y}+2 i \pi \theta\right) \psi_{n}\right)=\lambda_{n}(\theta) \rho_{0}(y) \psi_{n} \quad \text { in } \mathbb{T}^{N},
$$

corresponding to the $n$th eigenvalue or energy level $\lambda_{n}(\theta)$. As usual the interpretation of the Bloch parameter $\theta$ is that it is a reduced wave number and the eigenvalue is the square of a time frequency $\omega_{n}\left(\theta_{0}\right)$ defined by

$$
\omega_{n}\left(\theta_{0}\right)=\sqrt{\lambda_{n}\left(\theta_{0}\right)}
$$


The derivative of the frequency with respect to the wave number gives the group velocity

$$
\mathcal{V}=\frac{1}{2 \pi} \nabla \omega_{n}\left(\theta_{0}\right)=\frac{1}{4 \pi} \frac{1}{\sqrt{\lambda_{n}\left(\theta_{0}\right)}} \nabla \lambda_{n}\left(\theta_{0}\right)
$$

and the divergence of the group velocity yields a dispersion tensor

$$
A^{*}=\frac{1}{2 \pi} \operatorname{div}_{\theta} \mathcal{V}=\frac{1}{4 \pi^{2}} \nabla_{\theta} \nabla_{\theta} \omega_{n}\left(\theta_{0}\right)
$$

Our main assumptions is that $\lambda_{n}\left(\theta_{0}\right)$ is a simple eigenvalue and that the modulated coefficients $\rho_{1}$ and $A_{1}$ are "invariant along group lines", i.e.,

$$
\frac{\partial \rho_{1}}{\partial \tau} \pm \mathcal{V} \cdot \nabla_{x} \rho_{1}=0, \quad \frac{\partial A_{1}}{\partial \tau} \pm \mathcal{V} \cdot \nabla_{x} A_{1}=0
$$

In truth, we shall make a weaker but more technical assumption than (1.7) (see Sect. 5 for further details). We prove that, as $\varepsilon$ goes to 0 , the solution of (1.1) is asymptotically the sum of two wave packets

$$
u_{\varepsilon}(t, x) \approx \mathrm{e}^{2 i \pi \frac{\theta_{0} \cdot x}{\varepsilon}} \psi_{n}\left(\frac{x}{\varepsilon}, \theta_{0}\right)\left(\mathrm{e}^{i \frac{\omega_{n}\left(\theta_{0}\right) t}{\varepsilon^{2}}} v^{+}\left(t, x+\frac{\mathcal{V}}{\varepsilon} t\right)+\mathrm{e}^{-i \frac{\omega_{n}\left(\theta_{0}\right) t}{\varepsilon^{2}}} v^{-}\left(t, x-\frac{\mathcal{V}}{\varepsilon} t\right)\right),
$$

in a sense of weak two-scale convergence (see Theorem 5.1 and Remark 5.5). The envelope functions $v^{+}$and $v^{-}$, in the right-hand side of (1.8), are solutions of two Schrödinger equations, see (5.3) and (5.5). Each of them carries half of the initial data $v^{0}$ and opposite contributions in terms of the initial velocity $v^{1}$. The fact that the homogenized equations are of Schrödinger type was observed in the physics literature [24,29]. It is similar to the dispersive geometric optics of [14-16] and is reminiscent of the so-called parabolic or paraxial approximation for waves propagating in a privileged direction $[7,23,30]$.

Formula (1.8) yields a family of approximate travelling wave solutions of (1.1) with a coherent structure, even for long times. Remark that, when the group velocity $\mathcal{V}$ is zero (which happens, at least, at the bottom and top of each Bloch band), (1.8) is rather a stationary solution which is trapped by the periodic medium. As is well known there exists no propagating solution of the type of (1.8) with a frequency $\omega$ when $\omega^{2}$ is in a gap of the Bloch spectrum, i.e. when $\omega^{2} \neq \lambda_{n}(\theta)$ for all $n \geq 0$ and $\theta \in \mathbb{T}^{N}$. This property is a key feature of photonic crystals (see e.g. [8,22]). The fact that the homogenized equations for the envelope $v^{+}$and $v^{-}$are Schrödinger equations is a confirmation of the dispersive properties (i.e. the nonlinear character of the effective dispersion relation) of periodic composite materials as already studied in $[2,3,13,28]$.

We give a weak convergence proof of (1.8) (see Theorem 5.1) which is based on the notion of two-scale convergence with drift and on a simple, uniform in time, $L^{2}$ in space, estimate for the solution of (1.1). A strong convergence proof (for smooth coefficients), including the construction of infinite order asymptotic expansion of the solution, is given in a companion paper [5]. Note that, for technical reasons, the notations of the present paper and of [5] are not exactly the same.

The content of this paper is the following. Section 2 recalls the necessary tools of Bloch transform and two-scale convergence. Section 3 is concerned with the simpler case of purely periodic coefficients, i.e., $\rho_{1} \equiv 0$ and $A_{1} \equiv 0$ in (1.2). The asymptotic analysis is much simpler since it reduces to a Taylor expansion in the explicit formula for the solution in terms of Bloch waves. Section 4 is devoted to a priori estimates for (1.1) which are uniform with 
respect to $\varepsilon$. Section 5 contains our main homogenization results and their proofs. Section 6 makes a comparison with the well-known WKB method of geometric optics.

\section{Preliminaries}

In the present section we give our main assumptions, set some notations and a few preliminary results needed for stating and proving the main results of this paper.

We first assume that the coefficients $\rho_{0}(y)$ and $\left(A_{0}(y)\right)_{i j}$ are real, bounded and periodic functions, i.e. they belong to $L^{\infty}\left(\mathbb{T}^{N}\right)$, and that $\rho_{1}(t, \tau, x, y)$ and $\left(A_{1}(t, \tau, x, y)\right)_{i j}$, as well as their time derivatives $\frac{\partial \rho_{1}}{\partial t}$ and $\frac{\partial \rho_{1}}{\partial \tau}$ (same for $\left(A_{1}\right)_{i j}$ ), are bounded continuous functions, periodic with respect to $y$ (they could be merely Caratheodory functions, i.e., measurable with respect to $y$ and continuous in $t, \tau, x)$. In Sect. 5 we will make an additional assumption on $\rho_{1}$ and $A_{1}$ which we do not specify here.

Furthermore the density function $\rho_{0}$ is uniformly positive, i.e. there exists a positive constant $C>0$ such that $\rho_{0}(y) \geq C$ a.e. in $\mathbb{T}^{N}$, and the elastic tensor $A_{0}$ is symmetric, uniformly coercive, i.e. there exists another positive constant $C>0$ such that $A_{0}(y) \zeta \cdot \zeta \geq$ $C|\zeta|^{2}$ for any $\zeta \in \mathbb{R}^{N}$ and a.e. in $\mathbb{T}^{N}$.

Under these assumptions it is well-known $[9,12,21,27]$ that, for any value of the parameter $\theta \in \mathbb{T}^{N}$, the cell problem (1.4) defines a self-adjoint compact operator on $L^{2}\left(\mathbb{T}^{N}\right)$ (with the scalar product defined by $\langle u, v\rangle=\int_{\mathbb{T}^{N}} \rho_{0} u \bar{v} \mathrm{~d} y$ ) which admits a countable sequence of real increasing eigenvalues $\left\{\lambda_{n}\right\}_{n \geq 1}$ (repeated with their multiplicity) and orthonormalized eigenfunctions $\left\{\psi_{n}\right\}_{n \geq 1}$ with $\int_{\mathbb{T}^{N}} \rho_{0}\left|\psi_{n}\right|^{2} \mathrm{~d} y=1$. The dual parameter $\theta$ is called the Bloch frequency and it runs in the dual cell of $\mathbb{T}^{N}$, i.e. by periodicity it is enough to consider $\theta \in \mathbb{T}^{N}$.

Our main assumption is that there exist an energy level $n \geq 1$ and a Bloch parameter $\theta_{0} \in \mathbb{T}^{N}$ such that

$$
\lambda_{n}\left(\theta_{0}\right)>0 \text { is a simple eigenvalue. }
$$

Remark 2.1 We recall some basic facts about the Bloch spectral problem [9, 12,21,27]. The minimum of $\lambda_{1}(\theta)$ is zero and is uniquely attained at $\theta=0$ (this is a consequence of the maximum principle). Furthermore, the Hessian matrix at $\theta=0, \nabla_{\theta} \nabla_{\theta} \lambda_{1}(0)$, is positive definite since it is equal to the usual homogenized matrix for equation (1.1). In particular, it implies that there exists a positive constant $C>0$ such that $\lambda_{1}(\theta) \geq C|\theta|^{2}$. On the other hand, for any $n \geq 2$, there exists another positive constant $C>0$ such that $\min _{\theta} \lambda_{n}(\theta) \geq C>0$.

Remark 2.1 implies that $\lambda_{n}\left(\theta_{0}\right)>0$ except if $n=1$ and $\theta_{0}=0$. Therefore, the important part of assumption (2.1) is the simplicity of the eigenvalue. However, recall that simplicity is always generic, meaning that multiple eigenvalues are much more seldom than simple ones.

Under the simplicity assumption (2.1) it is a classical matter to prove that the $n$-th eigencouple of (1.4) is smooth in a neighborhood of $\theta_{0}$ (see e.g. [20]). To simplify the notations we introduce three operators, $\mathbb{A}_{n}(\theta), \mathbb{B}(\theta)$ and $\mathbb{C}(\theta)$, defined by

$$
\begin{gathered}
\mathbb{A}_{n}(\theta) \psi=-\left(\operatorname{div}_{y}+2 i \pi \theta\right)\left(A_{0}(y)\left(\nabla_{y}+2 i \pi \theta\right) \psi\right)-\lambda_{n}(\theta) \rho_{0}(y) \psi \quad \forall \psi \in L^{2}\left(\mathbb{T}^{N}\right), \\
\mathbb{B}(\theta) \psi=A_{0}(y)\left(\nabla_{y}+2 i \pi \theta\right) \psi \quad \forall \psi \in L^{2}\left(\mathbb{T}^{N}\right), \\
\mathbb{C}(\theta) \phi=\left(\operatorname{div}_{y}+2 i \pi \theta\right)\left(A_{0}(y) \phi\right) \quad \forall \phi \in L^{2}\left(\mathbb{T}^{N}\right)^{N} .
\end{gathered}
$$


Denoting by $\left(e_{k}\right)_{1 \leq k \leq N}$ the canonical basis of $\mathbb{R}^{N}$ and by $\left(\theta_{k}\right)_{1 \leq k \leq N}$ the components of $\theta$, the first derivative of (1.4) is

$$
\mathbb{A}_{n}(\theta) \frac{\partial \psi_{n}}{\partial \theta_{k}}=2 i \pi e_{k} \cdot \mathbb{B}(\theta) \psi_{n}+2 i \pi \mathbb{C}(\theta)\left(e_{k} \psi_{n}\right)+\frac{\partial \lambda_{n}}{\partial \theta_{k}}(\theta) \rho_{0}(y) \psi_{n},
$$

and its second derivative is

$$
\begin{aligned}
\mathbb{A}_{n}(\theta) \frac{\partial^{2} \psi_{n}}{\partial \theta_{k} \partial \theta_{l}}= & 2 i \pi e_{k} \cdot \mathbb{B}(\theta) \frac{\partial \psi_{n}}{\partial \theta_{l}}+2 i \pi \mathbb{C}(\theta)\left(e_{k} \frac{\partial \psi_{n}}{\partial \theta_{l}}\right)+\frac{\partial \lambda_{n}}{\partial \theta_{k}}(\theta) \rho_{0}(y) \frac{\partial \psi_{n}}{\partial \theta_{l}} \\
& +2 i \pi e_{l} \cdot \mathbb{B}(\theta) \frac{\partial \psi_{n}}{\partial \theta_{k}}+2 i \pi \mathbb{C}(\theta)\left(e_{l} \frac{\partial \psi_{n}}{\partial \theta_{k}}\right)+\frac{\partial \lambda_{n}}{\partial \theta_{l}}(\theta) \rho_{0}(y) \frac{\partial \psi_{n}}{\partial \theta_{k}} \\
& -4 \pi^{2} e_{k} A_{0}(y) e_{l} \psi_{n}-4 \pi^{2} e_{l} A_{0}(y) e_{k} \psi_{n}+\frac{\partial^{2} \lambda_{n}}{\partial \theta_{l} \partial \theta_{k}}(\theta) \rho_{0}(y) \psi_{n} .
\end{aligned}
$$

Multiplying (2.6) by $\bar{\psi}_{n}$, recalling the normalization of $\psi_{n}$ and integrating by parts we obtain

$$
\begin{aligned}
\frac{1}{4 \pi^{2}} \frac{\partial^{2} \lambda_{n}}{\partial \theta_{l} \partial \theta_{k}}(\theta)= & \int_{\mathbb{T}^{N}}\left(2\left|\psi_{n}\right|^{2} A_{0} e_{l} \cdot e_{k}-\pi^{-1} \omega_{n}(\theta) \rho_{0} \bar{\psi}_{n}\left(\mathcal{V}_{k} \frac{\partial \psi_{n}}{\partial \theta_{l}}+\mathcal{V}_{l} \frac{\partial \psi_{n}}{\partial \theta_{k}}\right)\right. \\
& +\frac{1}{2 i \pi}\left(\bar{\psi}_{n} \mathbb{B}(\theta) \frac{\partial \psi_{n}}{\partial \theta_{k}} \cdot e_{l}+\bar{\psi}_{n} \mathbb{B}(\theta) \frac{\partial \psi_{n}}{\partial \theta_{l}} \cdot e_{k}\right) \\
& \left.-\frac{1}{2 i \pi}\left(\frac{\partial \psi_{n}}{\partial \theta_{k}} \overline{\mathbb{B}(\theta) \psi_{n}} \cdot e_{l}+\frac{\partial \psi_{n}}{\partial \theta_{l}} \overline{\mathbb{B}(\theta) \psi_{n}} \cdot e_{k}\right)\right) \mathrm{d} y .
\end{aligned}
$$

We now give some results on the Bloch decomposition associated with the spectral problem (1.4) (see e.g. [9, 12]).

Lemma 2.2 Let $u(y), v(y) \in L^{2}\left(\mathbb{R}^{N}\right)$. Define their Bloch coefficients for $n \geq 1$ and $\theta \in \mathbb{T}^{N}$ $\alpha_{n}(\theta)=\int_{\mathbb{R}^{N}} \rho_{0}(y) u(y) \bar{\psi}_{n}(y, \theta) \mathrm{e}^{-2 i \pi \theta \cdot y} \mathrm{~d} y, \quad \beta_{n}(\theta)=\int_{\mathbb{R}^{N}} \rho_{0}(y) v(y) \bar{\psi}_{n}(y, \theta) \mathrm{e}^{-2 i \pi \theta \cdot y} \mathrm{~d} y$.

Then, $\alpha_{n}, \beta_{n}$ belong to $L^{2}\left(\mathbb{T}^{N}\right)$ and

$$
u(y)=\sum_{n \geq 1} \int_{\mathbb{T}^{N}} \alpha_{n}(\theta) \psi_{n}(y, \theta) e^{2 i \pi \theta \cdot y} \mathrm{~d} \theta, \quad v(y)=\sum_{n \geq 1} \int_{\mathbb{T}^{N}} \beta_{n}(\theta) \psi_{n}(y, \theta) e^{2 i \pi \theta \cdot y} \mathrm{~d} \theta,
$$

and they satisfy the Parseval equality

$$
\int_{\mathbb{R}^{N}} \rho_{0}(y) u(y) \bar{v}(y) \mathrm{d} y=\sum_{n \geq 1} \int_{\mathbb{T}^{N}} \alpha_{n}(\theta) \bar{\beta}_{n}(\theta) \mathrm{d} \theta .
$$

In other words, the Bloch transform $u \rightarrow\left\{\alpha_{n}(\theta)\right\}_{n \geq 1}$ is an isometry from $L^{2}\left(\mathbb{R}^{N}\right)$ into $\ell^{2}\left(L^{2}\left(\mathbb{T}^{N}\right)\right)$. Furthermore, the Bloch transform diagonalizes the elliptic operator in (1.1), in the sense that

$$
\int_{\mathbb{R}^{N}} A_{0}(y) \nabla u(y) \cdot \nabla \bar{v}(y) \mathrm{d} y=\sum_{n \geq 1} \int_{\mathbb{T}^{N}} \lambda_{n}(\theta) \alpha_{n}(\theta) \bar{\beta}_{n}(\theta) \mathrm{d} \theta .
$$

We recall the notion of two-scale convergence (see $[1,26])$. 
Proposition 2.3 Let $\left\{v_{\varepsilon}\right\}_{\varepsilon>0}$ be a bounded sequence in $L^{2}\left(\mathbb{R}^{N}\right)$. There exists a subsequence, still denoted by $\varepsilon$, and a limit $v^{*}(x, y) \in L^{2}\left(\mathbb{R}^{N} \times \mathbb{T}^{N}\right)$ such that $v_{\varepsilon}$ two-scale converges weakly to $v^{*}$ in the sense that

$$
\lim _{\varepsilon \rightarrow 0} \int_{\mathbb{R}^{N}} v_{\varepsilon}(x) \phi\left(x, \frac{x}{\varepsilon}\right) \mathrm{d} x=\int_{\mathbb{R}^{N}} \int_{\mathbb{T}^{N}} v^{*}(x, y) \phi(x, y) \mathrm{d} x \mathrm{~d} y
$$

for all functions $\phi(x, y) \in L^{2}\left(\mathbb{R}^{N} ; C\left(\mathbb{T}^{N}\right)\right)$ (i.e., $(0,1)^{N}$-periodic with respect to $y$ ).

In truth, in order to homogenize (1.1) we will need the following extension of the notion of two-scale convergence which was introduced in [25].

Proposition 2.4 Let $\mathcal{V} \in \mathbb{R}^{N}$ be a given drift velocity. Let $\left\{v_{\varepsilon}\right\}_{\varepsilon>0}$ be a uniformly bounded sequence in $L^{2}\left((0, T) \times \mathbb{R}^{N}\right)$. There exists a subsequence, still denoted by $\varepsilon$, and a limit function $v^{*}(t, x, y) \in L^{2}\left((0, T) \times \mathbb{R}^{N} \times \mathbb{T}^{N}\right)$ such that $v_{\varepsilon}$ two-scale converges weakly with drift to $v^{*}$ in the sense that

$$
\lim _{\varepsilon \rightarrow 0} \int_{0}^{T} \int_{\mathbb{R}^{N}} v_{\varepsilon}(t, x) \phi\left(t, x+\frac{\mathcal{V}}{\varepsilon} t, \frac{x}{\varepsilon}\right) \mathrm{d} t \mathrm{~d} x=\int_{0}^{T} \int_{\mathbb{R}^{N}} \int_{\mathbb{T}^{N}} v^{*}(t, x, y) \phi(t, x, y) \mathrm{d} t \mathrm{~d} x \mathrm{~d} y
$$

for all functions $\phi(t, x, y) \in L^{2}\left((0, T) \times \mathbb{R}^{N} ; C\left(\mathbb{T}^{N}\right)\right)$. Furthermore, if

$$
\lim _{\varepsilon \rightarrow 0}\left\|v_{\varepsilon}\right\|_{L^{2}\left((0, T) \times \mathbb{R}^{N}\right)}=\left\|v^{*}\right\|_{L^{2}\left((0, T) \times \mathbb{R}^{N} \times \mathbb{T}^{N}\right)},
$$

then the subsequence $v_{\varepsilon}$ two-scale converges strongly with drift to $v^{*}$ in the sense that, for any other sequence $w_{\varepsilon}$ which two-scale converges weakly with drift to $w^{*}(t, x, y) \in$ $L^{2}\left((0, T) \times \mathbb{R}^{N} \times \mathbb{T}^{N}\right)$, we have

$$
\lim _{\varepsilon \rightarrow 0} \int_{0}^{T} \int_{\mathbb{R}^{N}} v_{\varepsilon}(t, x) w_{\varepsilon}(t, x) \mathrm{d} t \mathrm{~d} x=\int_{0}^{T} \int_{\mathbb{R}^{N}} \int_{\mathbb{T}^{N}} v^{*}(t, x, y) w^{*}(t, x, y) \mathrm{d} t \mathrm{~d} x \mathrm{~d} y .
$$

Of course, the two-scale limit $v^{*}$ in Proposition 2.4 depends on the chosen drift velocity $\mathcal{V}$ but not on the final time $T$. We emphasize that, except when $\mathcal{V}=0$, Proposition 2.4 does not reduce to the usual definition of two-scale convergence upon the change of variable $z=x+\frac{\mathcal{V}}{\varepsilon} t$ because there is no drift in the fast variable $y=\frac{x}{\varepsilon}$.

In the sequel we shall need the following technical lemma which will be applied to the modulated coefficients $\rho_{1}, A_{1}$.

Lemma 2.5 Let a $(t, \tau, x, y)$ be a continuous bounded function on $\mathbb{R}^{+} \times \mathbb{R}^{+} \times \mathbb{R}^{N} \times \mathbb{T}^{N}$ such that $x \rightarrow \sup _{t, \tau, y}|a(t, \tau, x, y)|$ belongs to $L^{2}\left(\mathbb{R}^{N}\right)$, and which admits $a$ "weak average on group lines", i.e., there exists a function $a^{+}(t, x, y)$ such that

$$
\lim _{\mathcal{T} \rightarrow+\infty} \frac{1}{\mathcal{T}} \int_{0}^{\mathcal{T}} a(t, \tau, x-\mathcal{V} \tau, y) \mathrm{d} \tau=a^{+}(t, x, y),
$$

uniformly in ( $t, x, y)$. Then, $a\left(t, \frac{t}{\varepsilon}, x, \frac{x}{\varepsilon}\right)$ two-scale converges weakly with drift to $a^{+}(t, x, y)$. Furthermore, if a $(t, \tau, x, y)$ admits a "strong average on group lines", i.e., on top of (2.10) $a^{+}(t, x, y)$ satisfies also 


$$
\lim _{\mathcal{T} \rightarrow+\infty} \frac{1}{\mathcal{T}} \int_{0}^{\mathcal{T}} a^{2}(t, \tau, x-\mathcal{V} \tau, y) \mathrm{d} \tau=\left(a^{+}\right)^{2}(t, x, y)
$$

then $a\left(t, \frac{t}{\varepsilon}, x, \frac{x}{\varepsilon}\right)$ two-scale converges strongly with drift to $a^{+}(t, x, y)$.

Remark 2.6 In the sequel we shall assume that the modulated coefficients $\rho_{1}, A_{1}$ satisfy the hypothesis of "strong average on group lines" (2.10)-(2.11) with respect to both velocities $\pm \mathcal{V}$. Note that (2.10)-(2.11) is implied by the "-" equations in assumption (1.7) on "invariance along group lines": in such a case, we have $a(t, \tau, x, y)=a^{+}(t, x+\mathcal{V} \tau, y)$. In our companion paper [5] we make a different, slightly weaker, assumption on the modulated coefficients $\rho_{1}, A_{1}$ : we still assume $(2.10)$ but we replace $(2.11)$ by another assumption similar to (2.10) but involving more regularity of the coefficients.

Proof Under assumption (2.10) we compute the limit of

$$
\Delta_{\varepsilon}=\int_{0}^{T} \int_{\mathbb{R}^{N}} a\left(t, \frac{t}{\varepsilon}, x, \frac{x}{\varepsilon}\right) \phi\left(t, x+\frac{\mathcal{V}}{\varepsilon} t, \frac{x}{\varepsilon}\right) \mathrm{d} t \mathrm{~d} x
$$

for a smooth, compactly supported function $\phi(t, x, y) \in L^{2}\left((0, T) \times \mathbb{R}^{N} ; C\left(\mathbb{T}^{N}\right)\right)$. We first make the change of variables $x^{\prime}=x+\varepsilon^{-1} \mathcal{V} t$

$$
\Delta_{\varepsilon}=\int_{0}^{T} \int_{\mathbb{R}^{N}} a\left(t, \frac{t}{\varepsilon}, x^{\prime}-\varepsilon^{-1} \mathcal{V} t, \frac{x^{\prime}}{\varepsilon}-\varepsilon^{-2} \mathcal{V} t\right) \phi\left(t, x^{\prime}, \frac{x^{\prime}}{\varepsilon}-\varepsilon^{-2} \mathcal{V} t\right) \mathrm{d} t \mathrm{~d} x^{\prime}
$$

Let $\sigma>0$ and $\left\{m_{\varepsilon}\right\} \subset \mathbb{N}$ be a sequence of integer numbers such that $m_{\varepsilon} \varepsilon \rightarrow \sigma$. Introducing a regular paving $\mathbb{R}^{N}=\cup_{k \in \mathbb{Z}^{N}} Y_{k}^{\varepsilon}$ with $Y_{k}^{\varepsilon}=x_{k}^{\varepsilon}+\left(0, \varepsilon m_{\varepsilon}\right)^{N}$ and $x_{k}^{\varepsilon}=\varepsilon m_{\varepsilon} k$, using the continuity of $a$ and $\phi$, we deduce

$$
\Delta_{\varepsilon}=\sum_{k \in \mathbb{Z}^{N}}\left(\varepsilon m_{\varepsilon}\right)^{N} \int_{0}^{T} \int_{\mathbb{T}^{N}} a\left(t, \frac{t}{\varepsilon}, x_{k}^{\varepsilon}-\varepsilon^{-1} \mathcal{V} t, y\right) \phi\left(t, x_{k}^{\varepsilon}, y\right) \mathrm{d} t \mathrm{~d} y+\delta_{\varepsilon, \sigma}
$$

where $\delta_{\varepsilon, \sigma}$ denotes at various places different sequences of numbers going to zero as $\varepsilon, \sigma \rightarrow$ 0 . For a large positive integer $M$ we introduce a subdivision $0=t_{0}<t_{1}<\cdots<t_{M}=T$ such that, for all $0 \leq i \leq M-1$,

$$
\left\|a(t, \cdot, \cdot, \cdot)-a\left(t_{i}, \cdot, \cdot, \cdot\right)\right\|_{L^{\infty}\left(\mathbb{R}^{+}, \mathbb{R}^{N}, \mathbb{T}^{N}\right)} \leq \frac{1}{M} \quad \forall t \in\left[t_{i}, t_{i+1}\right]
$$

and the same for $\phi$. Then

$$
\Delta_{\varepsilon}=\sum_{i=0}^{M-1} \sum_{k \in \mathbb{Z}^{N}}\left(\varepsilon m_{\varepsilon}\right)^{N} \int_{\mathbb{T}^{N}} \phi\left(t_{i}, x_{k}^{\varepsilon}, y\right)\left(\int_{t_{i}}^{t_{i+1}} a\left(t_{i}, \frac{t}{\varepsilon}, x_{k}^{\varepsilon}-\varepsilon^{-1} \mathcal{V} t, y\right) \mathrm{d} t\right) \mathrm{d} y+\delta_{\varepsilon, \sigma} .
$$


For $\varepsilon$ small, the points $x_{k}^{\varepsilon}$ are close to $\sigma k$ and therefore, by continuity, we can replace $x_{k}^{\varepsilon}$ by $\sigma k$ in both $a$ and $\phi$, up to a small error. Then, introducing $s=\varepsilon^{-1} t$ we obtain, as $\varepsilon$ goes to 0 ,

$$
\begin{aligned}
& \int_{t_{i}}^{t_{i+1}} a\left(t_{i}, \frac{t}{\varepsilon}, \sigma k-\varepsilon^{-1} \mathcal{V} t, y\right) \mathrm{d} t \\
& \quad=\varepsilon \int_{0}^{\varepsilon^{-1} t_{i+1}} a\left(t_{i}, s, \sigma k-\mathcal{V} s, y\right) \mathrm{d} s-\varepsilon \int_{0}^{\varepsilon^{-1} t_{i}} a\left(t_{i}, s, \sigma k-\mathcal{V} s, y\right) \mathrm{d} s \\
& \rightarrow\left(t_{i+1}-t_{i}\right) a^{+}\left(t_{i}, \sigma k, y\right)
\end{aligned}
$$

by virtue of assumption (2.10). Consequently

$$
\Delta_{\varepsilon}=\sum_{i=0}^{M-1} \sum_{k \in \mathbb{Z}^{N}} \sigma^{N}\left(t_{i+1}-t_{i}\right) \int_{\mathbb{T}^{N}} \phi\left(t_{i}, \sigma k, y\right) a^{+}\left(t_{i}, \sigma k, y\right) \mathrm{d} y+\delta_{\varepsilon, \sigma}
$$

which, for $\varepsilon, \sigma \rightarrow 0$ and $M \rightarrow+\infty$, is just a Riemann sum approximation of

$$
\int_{0}^{T} \int_{\mathbb{R}^{N}} \int_{\mathbb{T}^{N}} a^{+}(t, x, y) \phi(t, x, y) \mathrm{d} t \mathrm{~d} x \mathrm{~d} y=\lim _{M \rightarrow+\infty} \lim _{\sigma \rightarrow 0} \lim _{\varepsilon \rightarrow 0} \Delta_{\varepsilon} .
$$

Therefore $a\left(t, \frac{t}{\varepsilon}, x, \frac{x}{\varepsilon}\right)$ two-scale converges weakly with drift to $a^{+}(t, x, y)$. The proof of the strong two-scale convergence, under assumption (2.11), is completely similar, so we safely leave it to the reader.

Notations The same letter $C$ denotes various positive constants which are all independent of $\varepsilon$ but whose precise value may change from place to place. Moreover by abuse of notation we write $\psi_{n}(y)$ and $\frac{\partial \psi_{n}}{\partial \theta}(y)$ to indicate the value of $\psi_{n}(y, \theta)$ and $\frac{\partial \psi_{n}}{\partial \theta}(y, \theta)$ respectively, evaluated at the point $\theta=\theta_{0}$ :

$$
\psi_{n}(y):=\psi_{n}\left(y, \theta_{0}\right), \quad \frac{\partial \psi_{n}}{\partial \theta}(y):=\frac{\partial \psi_{n}}{\partial \theta}\left(y, \theta_{0}\right) .
$$

Finally throughout this paper the Einstein summation convention is used.

\section{Explicit solutions in the periodic case}

In this section we restrict ourselves to the case of purely periodic coefficients, with no macroscopic modulations, and to special initial data defined as superposition of Bloch waves. Under those simplifying assumptions we obtain an explicit formula for the solution of the wave Eq. (1.1) on which, by means of a simple Taylor expansion, we can read off the homogenized equation. More precisely, we take $A_{1} \equiv 0$ and $\rho_{1} \equiv 0$ in (1.2) so that the coefficients are now purely periodic, i.e.

$$
A_{\varepsilon}(x)=A_{0}\left(\frac{x}{\varepsilon}\right), \quad \rho_{\varepsilon}(x)=\rho_{0}\left(\frac{x}{\varepsilon}\right) .
$$


On the other hand, instead of (1.3) we consider the following initial data

$$
\begin{aligned}
& u_{\varepsilon}^{0}(x)=\int_{\varepsilon^{-1} \mathbb{T}^{N}} \alpha(\eta) \psi_{n}\left(\frac{x}{\varepsilon}, \theta_{0}+\varepsilon \eta\right) \mathrm{e}^{2 i \pi \eta \cdot x+2 i \pi \frac{\theta_{0} \cdot x}{\varepsilon}} \mathrm{d} \eta, \\
& u_{\varepsilon}^{1}(x)=\varepsilon^{-2} \int_{\varepsilon^{-1} \mathbb{T}^{N}} \beta(\eta) \psi_{n}\left(\frac{x}{\varepsilon}, \theta_{0}+\varepsilon \eta\right) \mathrm{e}^{2 i \pi \eta \cdot x+2 i \pi \frac{\theta_{0} \cdot x}{\varepsilon}} \mathrm{d} \eta,
\end{aligned}
$$

where $\alpha(\eta)$ and $\beta(\eta)$ are smooth functions with compact support in $\mathbb{R}^{N}$. The advantage of (3.2) is twofold. First, upon the change of variables $\theta=\theta_{0}+\varepsilon \eta$, the initial data is already written as a Bloch decomposition (see Lemma 2.2) which is useful when we shall diagonalize the wave Eq. (1.1) by means of the Bloch transform. Second, thanks to the assumption on the compact support of $\alpha$ and $\beta$, the integrals on $\varepsilon^{-1} \mathbb{T}^{N}$ can be replaced by integrals on $\mathbb{R}^{N}$ (for sufficiently small $\varepsilon$ ) which yields a connection with the usual Fourier transform. Specifically, let us define the inverse Fourier transforms of $\alpha$ and $\beta$

$$
v^{0}(x)=\int_{\mathbb{R}^{N}} \alpha(\eta) \mathrm{e}^{2 i \pi \eta \cdot x} \mathrm{~d} \eta, \quad \text { and } \quad v^{1}(x)=\int_{\mathbb{R}^{N}} \beta(\eta) \mathrm{e}^{2 i \pi \eta \cdot x} \mathrm{~d} \eta,
$$

then, by a simple Taylor expansion of $\psi_{n}$ in (3.2), we deduce the following

Lemma 3.1 Under assumption (2.1) on the simplicity of $\lambda_{n}\left(\theta_{0}\right)$, we have

$$
\begin{gathered}
\left\|u_{\varepsilon}^{0}(x)-\psi_{n}\left(\frac{x}{\varepsilon}, \theta_{0}\right) \mathrm{e}^{2 i \pi \frac{\theta_{0} \cdot x}{\varepsilon}} v^{0}(x)\right\|_{L^{\infty}\left(\mathbb{R}^{N}\right)} \leq C \varepsilon, \\
\left\|\varepsilon^{2} u_{\varepsilon}^{1}(x)-\psi_{n}\left(\frac{x}{\varepsilon}, \theta_{0}\right) \mathrm{e}^{2 i \pi \frac{\theta_{0} \cdot x}{\varepsilon}} v^{1}(x)\right\|_{L^{\infty}\left(\mathbb{R}^{N}\right)} \leq C \varepsilon .
\end{gathered}
$$

Our main result in this section is

Theorem 3.2 Under the above assumptions and the simplicity hypothesis (2.1), the solution of the wave Eq. (1.1) is given by

$$
\begin{aligned}
u_{\varepsilon}(t, x)= & \mathrm{e}^{2 i \pi \frac{\theta_{0} \cdot x}{\varepsilon}} \psi_{n}\left(\frac{x}{\varepsilon}, \theta_{0}\right)\left(\mathrm{e}^{i \frac{\omega_{n}\left(\theta_{0}\right) t}{\varepsilon^{2}}} v^{+}\left(t, x+\frac{\mathcal{V}}{\varepsilon} t\right)\right. \\
& \left.+\mathrm{e}^{-i \frac{\omega_{n}\left(\theta_{0}\right) t}{\varepsilon^{2}}} v^{-}\left(t, x-\frac{\mathcal{V}}{\varepsilon} t\right)\right)+r_{\varepsilon}(t, x)
\end{aligned}
$$

with

$$
\left\|r_{\varepsilon}(t, x)\right\|_{\left.L^{\infty}\left((0, T) \times \mathbb{R}^{N}\right)\right)} \leq C \varepsilon,
$$

and $v^{ \pm} \in C\left([0, T] ; L^{2}\left(\mathbb{R}^{N}\right)\right)$ is the solution of the homogenized problem

$$
\begin{cases} \pm 2 i \frac{\partial v^{ \pm}}{\partial t}-\operatorname{div}\left(A^{*} \nabla v^{ \pm}\right)=0 & \text { in } \mathbb{R}^{N} \times(0, T), \\ v^{ \pm}(t=0, x)=\frac{1}{2}\left(v_{0}(x) \pm \frac{1}{i \omega_{n}\left(\theta_{0}\right)} v_{1}(x)\right) & \text { in } \mathbb{R}^{N}\end{cases}
$$

where $\omega_{n}\left(\theta_{0}\right)=\sqrt{\lambda_{n}\left(\theta_{0}\right)}, \mathcal{V}$ is the group velocity defined by (1.5) and $A^{*}$ is the homogenized dispersion tensor defined by (1.6). 
Proof Use the Bloch decomposition of Lemma 2.2 to diagonalize the elliptic operator in the wave Eq. (1.1). Write

$$
u_{\varepsilon}(t, x)=\sum_{k=1}^{+\infty} \int_{\varepsilon^{-1} \mathbb{T}^{N}} \alpha_{k}^{\varepsilon}(t, \eta) \psi_{k}\left(\frac{x}{\varepsilon}, \theta_{0}+\varepsilon \eta\right) \mathrm{e}^{2 i \pi \eta \cdot x+2 i \pi \frac{\theta_{0} \cdot x}{\varepsilon}} \mathrm{d} \eta,
$$

where the Bloch coefficients are determined as solutions of the initial value problems,

$$
\varepsilon^{2} \frac{\mathrm{d}^{2} \alpha_{k}^{\varepsilon}}{\mathrm{d} t^{2}}+\frac{\lambda_{k}\left(\theta_{0}+\varepsilon \eta\right)}{\varepsilon^{2}} \alpha_{k}^{\varepsilon}=0, \quad \alpha_{k}^{\varepsilon}(0)=\alpha(\eta) \delta_{k n}, \quad \frac{\mathrm{d} \alpha_{k}^{\varepsilon}}{\mathrm{d} t}(0)=\varepsilon^{-2} \beta(\eta) \delta_{k n},
$$

where $\delta_{k n}$ is the Kronecker symbol. For $k \neq n$ it follows that $\alpha_{k}^{\varepsilon} \equiv 0$. For $k=n$ the solution is equal to,

$$
\begin{aligned}
u_{\varepsilon}(t, x)= & \frac{1}{2} \int_{\varepsilon^{-1} \mathbb{T}^{N}}\left(\alpha(\eta)-\frac{i \beta(\eta)}{\omega_{n}\left(\theta_{0}+\varepsilon \eta\right)}\right) \psi_{n}\left(\frac{x}{\varepsilon}, \theta_{0}+\varepsilon \eta\right) \mathrm{e}^{2 i \pi \frac{\left(\theta_{0}+\varepsilon \eta\right) \cdot x}{\varepsilon}+i \frac{\omega_{n}\left(\theta_{0}+\varepsilon \eta\right)}{\varepsilon^{2}} t} \mathrm{~d} \eta \\
& +\frac{1}{2} \int_{\varepsilon^{-1} \mathbb{T}^{N}}\left(\alpha(\eta)+\frac{i \beta(\eta)}{\omega_{n}\left(\theta_{0}+\varepsilon \eta\right)}\right) \psi_{n}\left(\frac{x}{\varepsilon}, \theta_{0}+\varepsilon \eta\right) \mathrm{e}^{2 i \pi \frac{\left(\theta_{0}+\varepsilon \eta\right) \cdot x}{\varepsilon}-i \frac{\omega_{n}\left(\theta_{0}+\varepsilon \eta\right)}{\varepsilon^{2}} t} \mathrm{~d} \eta .
\end{aligned}
$$

Perform a Taylor expansion to second order to find,

$$
\begin{aligned}
\omega_{n}\left(\theta_{0}+\varepsilon \eta\right) & =\omega_{n}\left(\theta_{0}\right)+\nabla \omega_{n}\left(\theta_{0}\right) \cdot \varepsilon \eta+\frac{1}{2} \nabla \nabla \omega_{n}\left(\theta_{0}\right) \varepsilon^{2} \eta \cdot \eta+\mathcal{O}\left(\varepsilon^{3}\right) \\
& =\omega_{n}\left(\theta_{0}\right)+2 \pi \mathcal{V} \cdot \varepsilon \eta+2 \pi^{2} A^{*} \varepsilon^{2} \eta \cdot \eta+\mathcal{O}\left(\varepsilon^{3}\right) .
\end{aligned}
$$

Plug (3.7) into (3.6) and use a zero order Taylor expansion of $\psi_{n}$ to obtain

$$
\begin{aligned}
u_{\varepsilon}(t, x)= & \frac{1}{2} \psi_{n}\left(\frac{x}{\varepsilon}, \theta_{0}\right) \mathrm{e}^{2 i \pi \frac{\theta_{0} \cdot x}{\varepsilon}+i \frac{\omega_{n}\left(\theta_{0}\right)}{\varepsilon^{2}} t} \\
& \times \int_{\varepsilon^{-1} \mathbb{T}^{N}}\left(\alpha(\eta)-\frac{i \beta(\eta)}{\omega_{n}\left(\theta_{0}\right)}\right) \mathrm{e}^{2 i \pi\left(x+\frac{\mathcal{V}}{\varepsilon} t\right) \cdot \eta+2 i \pi^{2} A^{*} \eta \cdot \eta t+\mathcal{O}(\varepsilon) t} \mathrm{~d} \eta \\
& +\frac{1}{2} \psi_{n}\left(\frac{x}{\varepsilon}, \theta_{0}\right) \mathrm{e}^{2 i \pi \frac{\theta_{0} \cdot x}{\varepsilon}-i \frac{\omega_{n}\left(\theta_{0}\right)}{\varepsilon^{2}} t} \\
& \times \int_{\varepsilon^{-1} \mathbb{T}^{N}}\left(\alpha(\eta)+\frac{i \beta(\eta)}{\omega_{n}\left(\theta_{0}\right)}\right) \mathrm{e}^{2 i \pi\left(x-\frac{\mathcal{\nu}}{\varepsilon} t\right) \cdot \eta-2 i \pi^{2} A^{*} \eta \cdot \eta t+\mathcal{O}(\varepsilon) t} \mathrm{~d} \eta \\
& +R_{\varepsilon}(t, x)
\end{aligned}
$$

where $R_{\varepsilon}$ is the sum of higher order remainders, smaller than $\varepsilon$ in the $L^{\infty}$ norm. Since the functions $\alpha$ and $\beta$ are compactly supported, for $\varepsilon$ sufficiently small we can replace the integrals over $\varepsilon^{-1} \mathbb{T}^{N}$ by integrals over the whole space $\mathbb{R}^{N}$ and replace the factor $e^{\mathcal{O}(\varepsilon) t}$ by 1 since we consider finite times $0 \leq t \leq T$. To show that we obtain formula (3.3), consider the Fourier transform of the homogenized problem (3.4)

$$
\begin{cases} \pm 2 i \frac{\partial \hat{v}^{ \pm}}{\partial t}+4 \pi^{2} A^{*} \eta \cdot \eta \hat{v}^{ \pm}=0 & \text { in }(0, T) \times \mathbb{R}^{N}, \\ \hat{v}^{ \pm}(t=0, \eta)=\frac{1}{2}\left(\hat{v}_{0}(\eta) \pm \frac{1}{i \omega_{n}\left(\theta_{0}\right)} \hat{v}_{1}(\eta)\right) & \text { in } \mathbb{R}^{N} .\end{cases}
$$


The solution to (3.9) is given by

$$
\hat{v}^{ \pm}(t, x)=\frac{1}{2}\left(\alpha(\eta) \pm \frac{\beta(\eta)}{i \omega_{n}\left(\theta_{0}\right)}\right) \mathrm{e}^{ \pm 2 i \pi^{2} A^{*} \eta \cdot \eta t} .
$$

Therefore (3.8) can be rewritten as

$$
\begin{aligned}
u_{\varepsilon}(t, x)= & \psi_{n}\left(\frac{x}{\varepsilon}, \theta_{0}\right) \mathrm{e}^{2 i \pi \frac{\theta_{0} \cdot x}{\varepsilon}+i \frac{\omega_{n}\left(\theta_{0}\right) t}{\varepsilon^{2}}} \int_{\mathbb{R}^{N}} \hat{v}^{+}(t, x) \mathrm{e}^{2 i \pi\left(x+\frac{\mathcal{V}}{\varepsilon} t\right) \cdot \eta} \mathrm{d} \eta \\
& +\psi_{n}\left(\frac{x}{\varepsilon}, \theta_{0}\right) \mathrm{e}^{2 i \pi \frac{\theta_{0} \cdot x}{\varepsilon}-i \frac{\omega_{n}\left(\theta_{0}\right) t}{\varepsilon^{2}}} \int_{\mathbb{R}^{N}} \hat{v}^{-}(t, x) \mathrm{e}^{2 i \pi\left(x-\frac{\mathcal{V}}{\varepsilon} t\right) \cdot \eta} \mathrm{d} \eta+r_{\varepsilon}(t, x) \\
= & \mathrm{e}^{2 i \pi \frac{\theta_{0} \cdot x}{\varepsilon}} \psi_{n}\left(\frac{x}{\varepsilon}, \theta_{0}\right)\left(\mathrm{e}^{i \frac{\omega_{n}\left(\theta_{0}\right) t}{\varepsilon^{2}}} v^{+}\left(t, x+\frac{\mathcal{V}}{\varepsilon} t\right)+\mathrm{e}^{-i \frac{\omega_{n}\left(\theta_{0}\right) t}{\varepsilon^{2}}} v^{-}\left(t, x-\frac{\mathcal{V}}{\varepsilon} t\right)\right) \\
& +r_{\varepsilon}(t, x),
\end{aligned}
$$

where $r_{\varepsilon}$ takes into account the term $R_{\varepsilon}$ in (3.8) and the approximation we have done by replacing $\mathrm{e}^{\mathcal{O}(\varepsilon) t}$ by 1 , and is of order $\varepsilon$ in the $L^{\infty}$ norm.

Remark 3.3 If, as in (3.7), we expand $\psi_{n}$ to second order in $\varepsilon$ we construct correctors which could improve the error estimate. We obtain

$$
\begin{aligned}
u_{\varepsilon}(t, x) \approx & \mathrm{e}^{2 i \pi \frac{\theta_{0} \cdot x}{\varepsilon}+i \frac{\omega_{n}\left(\theta_{0}\right) t}{\varepsilon^{2}}}\left(\psi_{n}\left(\frac{x}{\varepsilon}\right)\left(v^{+}\right)^{\varepsilon}+\frac{\varepsilon}{2 i \pi} \sum_{k=1}^{N} \frac{\partial \psi_{n}}{\partial \theta_{k}}\left(\frac{x}{\varepsilon}\right)\left(\frac{\partial v^{+}}{\partial x_{k}}\right)^{\varepsilon}\right. \\
& \left.-\frac{\varepsilon^{2}}{4 \pi^{2}} \sum_{k, l=1}^{N} \frac{\partial^{2} \psi_{n}}{\partial \theta_{k} \partial \theta_{l}}\left(\frac{x}{\varepsilon}\right)\left(\frac{\partial^{2} v^{+}}{\partial x_{k} \partial x_{l}}\right)^{\varepsilon}\right) \\
& +\mathrm{e}^{2 i \pi \frac{\theta_{0} \cdot x}{\varepsilon}-i \frac{\omega_{n}\left(\theta_{0}\right) t}{\varepsilon^{2}}}\left(\psi_{n}\left(\frac{x}{\varepsilon}\right)\left(v^{-}\right)^{\varepsilon}+\frac{\varepsilon}{2 i \pi} \sum_{k=1}^{N} \frac{\partial \psi_{n}}{\partial \theta_{k}}\left(\frac{x}{\varepsilon}\right)\left(\frac{\partial v^{-}}{\partial x_{k}}\right)^{\varepsilon}\right. \\
& \left.-\frac{\varepsilon^{2}}{4 \pi^{2}} \sum_{k, l=1}^{N} \frac{\partial^{2} \psi_{n}}{\partial \theta_{k} \partial \theta_{l}}\left(\frac{x}{\varepsilon}\right)\left(\frac{\partial^{2} v^{-}}{\partial x_{k} \partial x_{l}}\right)^{\varepsilon}\right) .
\end{aligned}
$$

In (3.10) we use the notation

$$
\left(v^{+}\right)^{\varepsilon}:=v^{+}\left(t, x+\frac{\mathcal{V}}{\varepsilon} t\right), \quad\left(v^{-}\right)^{\varepsilon}:=v^{-}\left(t, x-\frac{\mathcal{V}}{\varepsilon} t\right),
$$

and similarly for their derivatives. However, to get an error of order $\mathcal{O}\left(\varepsilon^{3}\right)$ in the $L^{\infty}((0, T) \times$ $\mathbb{R}^{N}$ ) norm in (3.10), we also need to perform a second order expansion of the remainder $e^{\mathcal{O}(\varepsilon) t}$ in the integrand of (3.8) which would add new differential terms with coefficients of order $\varepsilon$ and $\varepsilon^{2}$ in the homogenized equation (3.4). Equation (3.10) is the beginning of a two scale asymptotic expansion of $u_{\varepsilon}$. An infinite order asymptotic expansion of $u_{\varepsilon}$ is performed in our companion paper [5].

\section{A priori estimate}

We now come back to the general case of oscillating coefficients which are macroscopically modulated, as defined in (1.2). The goal of this section is to obtain a uniform a priori 
estimate for the solutions of equation (1.1). Remark that uniform bounds for (1.1) are not completely obvious, neither standard, because of the time scaling. Moreover observe that, although we do not require any positivity assumption on the coefficients $\rho_{1}$ and $A_{1}$, for each $\varepsilon$ sufficiently small the existence and uniqueness of the solution $u_{\varepsilon}$ in $C\left([0, T] ; H^{1}\left(\mathbb{R}^{N}\right)\right) \cap$ $C^{1}\left([0, T] ; L^{2}\left(\mathbb{R}^{N}\right)\right)$ is ensured by the positivity assumption on $\rho_{0}$ and $A_{0}$ together with the assumption of boundedness of $\rho_{1}, A_{1}$ and their time derivatives.

Proposition 4.1 The solution $u_{\varepsilon}$ of (1.1) satisfies

$$
\varepsilon\left\|\nabla u_{\varepsilon}\right\|_{L^{\infty}\left((0, T) ; L^{2}\left(\mathbb{R}^{N}\right)^{N}\right)}+\varepsilon^{2}\left\|\frac{\partial u_{\varepsilon}}{\partial t}\right\|_{L^{\infty}\left((0, T) ; L^{2}\left(\mathbb{R}^{N}\right)\right)} \leq C\left(\left\|v^{0}\right\|_{H^{1}\left(\mathbb{R}^{N}\right)}+\left\|v^{1}\right\|_{L^{2}\left(\mathbb{R}^{N}\right)}\right),
$$

and

$$
\left\|u_{\varepsilon}\right\|_{L^{\infty}\left((0, T) ; L^{2}\left(\mathbb{R}^{N}\right)\right)} \leq C\left(\left\|v^{0}\right\|_{H^{1}\left(\mathbb{R}^{N}\right)}+\left\|v^{1}\right\|_{H^{2}\left(\mathbb{R}^{N}\right)}\right),
$$

where $C(T)>0$ is a constant which does not depend on $\varepsilon$.

Proof In a first step we multiply the wave equation (1.1) by $\frac{\partial \bar{u}_{\varepsilon}}{\partial t}$ and take the real part to obtain an energy conservation which is not exact because the coefficients depend on time. Introducing the energy

$$
E\left(u_{\varepsilon}(t)\right)=\frac{1}{2} \int_{\mathbb{R}^{N}}\left(\varepsilon^{2} \rho_{\varepsilon}\left|\frac{\partial u_{\varepsilon}}{\partial t}\right|^{2}+A_{\varepsilon} \nabla u_{\varepsilon} \cdot \nabla \bar{u}_{\varepsilon}\right) \mathrm{d} x
$$

we obtain

$$
E\left(u_{\varepsilon}(t)\right)=E\left(u_{\varepsilon}(0)\right)+\frac{\varepsilon^{2}}{2} \int_{0}^{t} \int_{\mathbb{R}^{N}}\left(-\varepsilon^{2} \frac{\partial \rho_{1, \varepsilon}}{\partial t}\left|\frac{\partial u_{\varepsilon}}{\partial t}\right|^{2}+\frac{\partial A_{1, \varepsilon}}{\partial t} \nabla u_{\varepsilon} \cdot \nabla \bar{u}_{\varepsilon}\right) \mathrm{d} s \mathrm{~d} x,
$$

where $\rho_{1, \varepsilon}(t, x)=\rho_{1}\left(t, \frac{t}{\varepsilon}, x, \frac{x}{\varepsilon}\right)$ (same for $A_{1, \varepsilon}$ ). Here and in the sequel, for the sake of notational simplicity, we adopt the convention that

$$
\frac{\partial \rho_{1, \varepsilon}}{\partial t}:=\frac{\partial}{\partial t}\left(\rho_{1}\left(t, \frac{t}{\varepsilon}, x, \frac{x}{\varepsilon}\right)\right)=\left(\frac{\partial \rho_{1}}{\partial t}+\varepsilon^{-1} \frac{\partial \rho_{1}}{\partial \tau}\right)\left(t, \frac{t}{\varepsilon}, x, \frac{x}{\varepsilon}\right) .
$$

Thus, the time derivatives of $\rho_{1, \varepsilon}$ and $A_{1, \varepsilon}$ are of order $\varepsilon^{-1}$, and the dissipative term in the energy balance (4.4) is bounded by

$$
C T \varepsilon \max _{0 \leq t \leq T} E\left(u_{\varepsilon}(t)\right),
$$

which, for small $\varepsilon$, implies

$$
E\left(u_{\varepsilon}(t)\right) \leq C E\left(u_{\varepsilon}(0)\right) .
$$

Because of our choice of initial data (1.3), we deduce (4.1). To obtain (4.2) we use a classical regularization trick, namely we define

$$
w_{\varepsilon}(t, x)=\mathrm{e}^{-\gamma t}\left(\int_{0}^{t} \mathrm{e}^{\gamma s} u_{\varepsilon}(s, x) \mathrm{d} s+\chi_{\varepsilon}(x)\right),
$$


where $\chi_{\varepsilon}$ is defined as the unique solution in $H^{1}\left(\mathbb{R}^{N}\right)$ of

$$
-\operatorname{div}\left(A_{\varepsilon}(0) \nabla \chi_{\varepsilon}\right)+\gamma^{2} \varepsilon^{2} \rho_{\varepsilon}(0) \chi_{\varepsilon}=-\varepsilon^{2} \rho_{\varepsilon}(0) u_{\varepsilon}^{1}+\gamma \varepsilon^{2} \rho_{\varepsilon}(0) u_{\varepsilon}^{0} \quad \text { in } \mathbb{R}^{N} .
$$

The time exponentials in the definition of $w_{\varepsilon}$ yield a zero-order term in (4.6), when $\gamma \neq 0$, which makes the analysis easier. In the sequel it is enough to take $\gamma=1$. The notations $A_{\varepsilon}(0)$ and $\rho_{\varepsilon}(0)$ mean that these coefficients are evaluated at time $t=0$. In particular it implies that $\chi_{\varepsilon}$ does not depend on time. By definition of $w_{\varepsilon}$, one finds

$$
\begin{aligned}
\int_{0}^{t} \mathrm{e}^{\gamma s} \operatorname{div}\left(A_{\varepsilon} \nabla u_{\varepsilon}\right) \mathrm{d} s= & \int_{0}^{t} \operatorname{div}\left(A_{\varepsilon} \nabla \frac{\partial}{\partial t}\left(\mathrm{e}^{\gamma s} w_{\varepsilon}\right)\right) \mathrm{d} s \\
= & -\int_{0}^{t} \operatorname{div}\left(\frac{\partial A_{\varepsilon}}{\partial t} \nabla\left(\mathrm{e}^{\gamma s} w_{\varepsilon}\right)\right) \mathrm{d} s+\mathrm{e}^{\gamma t} \operatorname{div}\left(A_{\varepsilon} \nabla w_{\varepsilon}\right)(t) \\
& -\operatorname{div}\left(A_{\varepsilon}(0) \nabla \chi_{\varepsilon}\right) .
\end{aligned}
$$

On the other hand, using (1.1) we write

$$
\begin{aligned}
\int_{0}^{t} \varepsilon^{-2} \mathrm{e}^{\gamma s} \operatorname{div}\left(A_{\varepsilon} \nabla u_{\varepsilon}\right) \mathrm{d} s= & \int_{0}^{t} \mathrm{e}^{\gamma s} \frac{\partial}{\partial t}\left(\rho_{\varepsilon} \frac{\partial u_{\varepsilon}}{\partial t}\right) \mathrm{d} s \\
= & \mathrm{e}^{\gamma t}\left(\frac{\partial}{\partial t}\left(\rho_{\varepsilon} \frac{\partial w_{\varepsilon}}{\partial t}\right)-\gamma^{2} \rho_{\varepsilon} w_{\varepsilon}\right) \\
& -\mathrm{e}^{\gamma t} \frac{\partial \rho_{\varepsilon}}{\partial t} \frac{\partial w_{\varepsilon}}{\partial t}+\rho_{\varepsilon}(0)\left(\gamma u_{\varepsilon}^{0}-u_{\varepsilon}^{1}\right) \\
& +\gamma \int_{0}^{t} \mathrm{e}^{\gamma s}\left(\frac{\partial \rho_{\varepsilon}}{\partial t}+\gamma \rho_{\varepsilon}\right) u_{\varepsilon} \mathrm{d} s
\end{aligned}
$$

Then, combining (4.7) and (4.8), a lengthy but simple computation shows that $w_{\varepsilon}$ is the unique solution of the same wave equation with different initial data and a source term

$$
\begin{cases}\varepsilon^{2} \frac{\partial}{\partial t}\left(\rho_{\varepsilon} \frac{\partial w_{\varepsilon}}{\partial t}\right)-\operatorname{div}\left(A_{\varepsilon} \nabla w_{\varepsilon}\right)=f_{\varepsilon} & \text { in } \mathbb{R}^{N} \times(0, T), \\ w_{\varepsilon}(0, x)=\chi_{\varepsilon}(x) & \text { in } \mathbb{R}^{N}, \\ \frac{\partial w_{\varepsilon}}{\partial t}(0, x)=u_{\varepsilon}^{0}(x)-\gamma \chi_{\varepsilon}(x) & \text { in } \mathbb{R}^{N},\end{cases}
$$

where the source term is

$$
f_{\varepsilon}(t, x)=\varepsilon^{4} \frac{\partial \rho_{1, \varepsilon}}{\partial t} \frac{\partial w_{\varepsilon}}{\partial t}-\varepsilon^{2} \int_{0}^{t} \mathrm{e}^{\gamma(s-t)}\left(\varepsilon^{2} \gamma \frac{\partial \rho_{1, \varepsilon}}{\partial t} \frac{\partial w_{\varepsilon}}{\partial t}+\operatorname{div}\left(\frac{\partial A_{1, \varepsilon}}{\partial t} \nabla w_{\varepsilon}\right)\right)(s) \mathrm{d} s .
$$

Remark that this source term vanishes if the coefficients do not depend on time. We write the energy conservation for $w_{\varepsilon}$

$$
\begin{aligned}
E\left(w_{\varepsilon}(t)\right)= & E\left(w_{\varepsilon}(0)\right)+\int_{0}^{t} \int_{\mathbb{R}^{N}} f_{\varepsilon} \frac{\partial w_{\varepsilon}}{\partial t} \mathrm{~d} t \mathrm{~d} x \\
& +\frac{\varepsilon^{2}}{2} \int_{0}^{t} \int_{\mathbb{R}^{N}}\left(-\varepsilon^{2} \frac{\partial \rho_{1, \varepsilon}}{\partial t}\left|\frac{\partial w_{\varepsilon}}{\partial t}\right|^{2}+\frac{\partial A_{1, \varepsilon}}{\partial t} \nabla w_{\varepsilon} \cdot \nabla \bar{w}_{\varepsilon}\right) \mathrm{d} t \mathrm{~d} x,
\end{aligned}
$$


with

$$
E\left(w_{\varepsilon}(0)\right)=\frac{1}{2} \int_{\mathbb{R}^{N}}\left(\varepsilon^{2} \rho_{\varepsilon}\left|u_{\varepsilon}^{0}-\gamma \chi_{\varepsilon}\right|^{2}+A_{\varepsilon} \nabla \chi_{\varepsilon} \cdot \nabla \bar{\chi}_{\varepsilon}\right) \mathrm{d} x .
$$

As in the energy balance (4.4) for $u_{\varepsilon}$, the dissipative term on the last line of (4.10) is bounded by $C T \varepsilon \max _{0 \leq t \leq T} E\left(w_{\varepsilon}(t)\right)$. In order to estimate the other dissipative term due to the source term, we first compute its part involving $A_{1, \varepsilon}$ by two integrations by parts in $t$ and $x$

$$
\begin{aligned}
& -\varepsilon^{2} \int_{0}^{T} \int_{\mathbb{R}^{N}} \frac{\partial w_{\varepsilon}}{\partial t}(t) \int_{0}^{t} e^{\gamma(s-t)} \mathrm{div}\left(\frac{\partial A_{1, \varepsilon}}{\partial t} \nabla w_{\varepsilon}\right)(s) \mathrm{d} s \mathrm{~d} t \mathrm{~d} x \\
& =-\varepsilon^{2} \int_{0}^{T} \int_{\mathbb{R}^{N}} \frac{\partial A_{1, \varepsilon}}{\partial t} \nabla w_{\varepsilon} \cdot \nabla\left(w_{\varepsilon}-\mathrm{e}^{\gamma(t-T)} w_{\varepsilon}(T)\right) \mathrm{d} t \mathrm{~d} x \\
& +\gamma \varepsilon^{2} \int_{0}^{T} \int_{\mathbb{R}^{N}} \int_{0}^{t} \mathrm{e}^{\gamma(s-t)} \frac{\partial A_{1, \varepsilon}}{\partial t}(s) \nabla w_{\varepsilon}(s) \cdot \nabla w_{\varepsilon}(t) \mathrm{d} s \mathrm{~d} t \mathrm{~d} x
\end{aligned}
$$

which is again bounded in absolute value by $C T \varepsilon \max _{0 \leq t \leq T} E\left(w_{\varepsilon}(t)\right)$ because $\frac{\partial A_{1, \varepsilon}}{\partial t}$ is uniformly bounded by $C \varepsilon^{-1}$. The other terms involving the time derivative of $\rho_{1, \varepsilon}$ in the definition of $f_{\varepsilon}$ are easy to bound and we obtain the following estimate

$$
\left|\int_{0}^{t} \int_{\mathbb{R}^{N}} f_{\varepsilon} \frac{\partial w_{\varepsilon}}{\partial t} \mathrm{~d} t \mathrm{~d} x\right| \leq C T \varepsilon\left(\max _{0 \leq t \leq T} E\left(w_{\varepsilon}(t)\right)+\varepsilon^{2}\left\|\frac{\partial w_{\varepsilon}}{\partial t}\right\|_{L^{\infty}\left((0, T) ; L^{2}\left(\mathbb{R}^{N}\right)\right)}^{2}\right) .
$$

Thanks to Proposition 4.2 below we have $E\left(w_{\varepsilon}(0)\right)=\mathcal{O}\left(\varepsilon^{2}\right)$. Thus, we deduce that $E\left(w_{\varepsilon}(t)\right)=\mathcal{O}\left(\varepsilon^{2}\right)$ and

$$
\left\|\frac{\partial w_{\varepsilon}}{\partial t}\right\|_{L^{\infty}\left((0, T) ; L^{2}\left(\mathbb{R}^{N}\right)\right)} \leq C .
$$

Since $w_{\varepsilon}(0)=\chi_{\varepsilon}$ which is bounded in $L^{2}\left(\mathbb{R}^{N}\right)$, we also deduce

$$
\left\|w_{\varepsilon}\right\|_{L^{\infty}\left((0, T) ; L^{2}\left(\mathbb{R}^{N}\right)\right)} \leq C .
$$

Finally, by definition of $w_{\varepsilon}$ we have

$$
u_{\varepsilon}=\frac{\partial w_{\varepsilon}}{\partial t}+\gamma w_{\varepsilon}
$$

which implies (4.2).

Proposition 4.2 For $\gamma \neq 0$ the solution $\chi_{\varepsilon}$ of (4.6) satisfies

$$
\left\|\chi_{\varepsilon}\right\|_{L^{2}\left(\mathbb{R}^{N}\right)}+\varepsilon^{-1}\left\|\nabla \chi_{\varepsilon}\right\|_{L^{2}\left(\mathbb{R}^{N}\right)^{N}} \leq C .
$$

Remark 4.3 While the estimate on $\left\|\nabla \chi_{\varepsilon}\right\|_{L^{2}\left(\mathbb{R}^{N}\right)^{N}}$ provided by Proposition 4.2 is optimal, that on $\left\|\chi_{\varepsilon}\right\|_{L^{2}\left(\mathbb{R}^{N}\right)}$ is too pessimistic. Indeed, by formal two-scale asymptotic expansions it is easily seen that

$$
\chi_{\varepsilon}(x) \approx \frac{-\varepsilon^{2}}{\lambda_{n}\left(\theta_{0}\right)} \mathrm{e}^{2 i \pi \frac{\theta_{0} \cdot x}{\varepsilon}} \psi_{n}\left(\frac{x}{\varepsilon}\right) v_{1}(x)
$$


Therefore we expect that the optimal estimate is $\left\|\chi_{\varepsilon}\right\|_{L^{2}\left(\mathbb{R}^{N}\right)} \leq C \varepsilon^{2}$. A proof of this can be obtained by comparison between $\chi_{\varepsilon}$ and a truncated two-scale asymptotic expansion of it. However, such a proof is tedious since it requires at least four terms, i.e. up to the $\varepsilon^{3}$ term. We skip it since it is not necessary for the sequel.

Proof of Proposition 4.2 Recall that, by assumption (2.1), we have $\lambda_{n}\left(\theta_{0}\right)>0$ which implies that, either $\theta_{0} \neq 0$, or $n \geq 2$ if $\theta_{0}=0$ (since the only forbidden case is $\lambda_{1}(0)=0$ ). Multiplying (4.6) by $\chi_{\varepsilon}$, integrating by parts, using the coercivity assumption on the coefficients and the definition (1.3) of the initial data yields

$$
\left\|\nabla \chi_{\varepsilon}\right\|_{L^{2}\left(\mathbb{R}^{N}\right)}^{2}+\varepsilon^{2}\left\|\chi_{\varepsilon}\right\|_{L^{2}\left(\mathbb{R}^{N}\right)}^{2} \leq C\left|\int_{\mathbb{R}^{N}} \rho_{\varepsilon} \psi_{n}^{\varepsilon} \mathrm{e}^{2 i \pi \frac{\theta_{0} \cdot x}{\varepsilon}}\left(v_{1}-\varepsilon^{2} v_{0}\right) \bar{\chi}_{\varepsilon} \mathrm{d} x\right| .
$$

Let us prove that

$$
\left|\int_{\mathbb{R}^{N}} \rho_{0}\left(\frac{x}{\varepsilon}\right) \psi_{n}^{\varepsilon} \mathrm{e}^{2 i \pi \frac{\theta_{0} \cdot x}{\varepsilon}} v_{1} \bar{\chi}_{\varepsilon} \mathrm{d} x\right| \leq C \varepsilon\left\|v^{1}\right\|_{H^{2}\left(\mathbb{R}^{N}\right)}\left(\left\|\nabla \chi_{\varepsilon}\right\|_{L^{2}\left(\mathbb{R}^{N}\right)}+\varepsilon\left\|\chi_{\varepsilon}\right\|_{L^{2}\left(\mathbb{R}^{N}\right)}\right)
$$

which clearly implies the desired result.

First case: assume that $\theta_{0} \neq 0$. By Lemma 4.4 there exists $V \in L^{2}\left(\mathbb{T}^{N}\right)^{N}$ such that

$$
\begin{aligned}
-\operatorname{div}\left(V \mathrm{e}^{2 i \pi \theta_{0} \cdot y}\right) & =\rho_{0} \psi_{n} \mathrm{e}^{2 i \pi \theta_{0} \cdot y} \quad \forall \theta \in \mathbb{T}^{N}, \\
\|V\|_{L^{2}\left(\mathbb{T}^{N}\right)^{N}} & \leq C\left\|\rho_{0} \psi_{n}\right\|_{L^{2}\left(\mathbb{T}^{N}\right)} .
\end{aligned}
$$

Therefore after integrating by parts we can rewrite the left-hand side of (4.12) as follows

$$
\varepsilon \int_{\mathbb{R}^{N}} V\left(\frac{x}{\varepsilon}\right) \mathrm{e}^{2 i \pi \frac{\theta_{0} \cdot x}{\varepsilon}} \cdot\left(v_{1} \nabla \bar{\chi}_{\varepsilon}+\bar{\chi}_{\varepsilon} \nabla v_{1}\right) \mathrm{d} x .
$$

We can apply once more Lemma 4.4 to the second term in (4.13) to obtain

$$
\varepsilon^{2} \int_{\mathbb{R}^{N}} W\left(\frac{x}{\varepsilon}\right) \mathrm{e}^{2 i \pi \frac{\theta_{0} \cdot x}{\varepsilon}} \cdot \nabla\left(\bar{\chi}_{\varepsilon} \nabla v_{1}\right) \mathrm{d} x
$$

where $W$ is a matrix-valued function with entries in $L^{\infty}\left(\mathbb{T}^{N}\right)$. Since $V$ and $W$ are bounded functions, Cauchy-Schwarz inequality then leads to (4.12).

Second case: assume now that $\theta_{0}=0$ and $n \geq 2$. Since $\lambda_{1}(0)=0$ and $\psi_{1}(y, 0) \equiv 1$, by orthogonality of the eigenfunctions we deduce

$$
\int_{\mathbb{T}^{N}} \rho_{0}(y) \psi_{n}(y, 0) \mathrm{d} y=0 .
$$

Therefore we can still apply Lemma 4.4 and a calculation completely similar to that in the first case yields the same result. This concludes the proof of Proposition 4.2.

We conclude this section with a technical lemma on the divergence of Bloch wave vector fields that was used in the proof of Proposition 4.2. 
Lemma 4.4 For any $\eta \neq 0 \in \mathbb{T}^{N}$, there exists a positive constant $C(\eta)>0$ such that, for any $\varphi \in L^{2}\left(\mathbb{T}^{N}\right)$, there exists a (non unique) vector field $V \in L^{2}\left(\mathbb{T}^{N}\right)^{N}$ such that

$$
\begin{aligned}
-\operatorname{div}\left(V(y) \mathrm{e}^{2 i \pi \eta \cdot y}\right) & =\varphi(y) \mathrm{e}^{2 i \pi \eta \cdot y} \quad \text { in } \mathbb{T}^{N}, \\
\|V\|_{L^{2}\left(\mathbb{T}^{N}\right)} & \leq C(\eta)\|\varphi\|_{L^{2}\left(\mathbb{T}^{N}\right)} .
\end{aligned}
$$

When $\eta=0$ the same result holds true provided that $\int_{\mathbb{T}^{N}} \varphi d y=0$, and in such a case one can choose the vector field $V$ such that $\int_{\mathbb{T}^{N}} V \mathrm{~d} y=0$.

Furthermore, if $\varphi \in L^{\infty}\left(\mathbb{T}^{N}\right)$, then the vector field $V$ is continuous in $\mathbb{T}^{N}$.

Proof For $\eta=0$ this is a classical result. For $\eta \neq 0$, we introduce the unique solution $\chi \in H^{1}\left(\mathbb{T}^{N}\right)$ of

$$
-(\operatorname{div}+2 i \pi \eta)(\nabla+2 i \pi \eta) \chi=\varphi,
$$

and we define $V:=(\nabla+2 i \pi \eta) \chi$ which solves (4.14). If $\varphi \in L^{\infty}\left(\mathbb{T}^{N}\right)$, then $\chi \in W^{2, p}\left(\mathbb{T}^{N}\right)$ for any finite $p$ and, by Sobolev embeddings, $V$ is continuous in $\mathbb{T}^{N}$.

\section{Homogenization}

Recall that the time frequency $\omega$ is related to the reduced wave number (or Bloch parameter) $\theta$ by the so-called dispersion relation $\omega\left(\theta_{0}\right)=\sqrt{\lambda_{n}\left(\theta_{0}\right)}$. Then, the group velocity $\mathcal{V}$ is defined by (1.5) as $\nabla_{\theta} \omega\left(\theta_{0}\right) / 2 \pi$. Our precise assumptions on $\rho_{1}(t, \tau, x, y)$ and $A_{1}(t, \tau, x, y)$ are that they satisfy the hypothesis of "strong average along the group lines" (2.10) and (2.11) with respect to both velocities $\pm \mathcal{V}$. In other words, we assume that there exist $\rho_{1}^{ \pm}(t, x, y)$ and $A_{1}^{ \pm}(t, x, y)$ such that, for $a=\rho_{1}$ or $A_{1}$,

$$
\lim _{\mathcal{T} \rightarrow+\infty} \frac{1}{\mathcal{T}} \int_{0}^{\mathcal{T}}\left\{a, a^{2}\right\}(t, \tau, x-( \pm \mathcal{V}) \tau, y) \mathrm{d} \tau=\left\{a^{ \pm},\left(a^{ \pm}\right)^{2}\right\}(t, x, y),
$$

uniformly in $(t, x, y)$ (see Remark 2.6 for comments on this assumption). We then introduce two homogenized coefficients defined by

$$
\gamma^{ \pm}(t, x)=\int_{\mathbb{T}^{N}}\left(A_{1}^{ \pm}(t, x, y) \nabla \psi_{n}(y) \cdot \nabla \bar{\psi}_{n}(y)-\lambda_{n}\left(\theta_{0}\right) \rho_{1}^{ \pm}(t, x, y)\left|\psi_{n}(y)\right|^{2}\right) \mathrm{d} y .
$$

Theorem 5.1 Assume that (2.1) holds true (i.e., $\lambda_{n}\left(\theta_{0}\right)$ is a simple eigenvalue) and that the initial data are given by (1.3) with $v_{0} \in H^{1}\left(\mathbb{R}^{N}\right)$ and $v_{1} \in H^{2}\left(\mathbb{R}^{N}\right)$. Then the solution of (1.1) can be written as

$$
u_{\varepsilon}(t, x)=\mathrm{e}^{i \frac{\omega_{n}\left(\theta_{0}\right) t}{\varepsilon^{2}}} \mathrm{e}^{2 i \pi \frac{\theta_{0} \cdot x}{\varepsilon}} v_{\varepsilon}^{+}(t, x)
$$

where $v_{\varepsilon}^{+}$two-scale converges with drift $\mathcal{V}$ to $\psi_{n}\left(y, \theta_{0}\right) v^{+}(t, x)$ and $v^{+} \in C\left([0, T] ; L^{2}\left(\mathbb{R}^{N}\right)\right)$ is the solution of the homogenized problem

$$
\begin{cases}2 i \frac{\partial v^{+}}{\partial t}-\operatorname{div}\left(A^{*} \nabla v^{+}\right)+\frac{\gamma^{+}}{\omega_{n}\left(\theta_{0}\right)} v^{+}=0 & \text { in } \mathbb{R}^{N} \times(0, T), \\ v^{+}(t=0, x)=\frac{1}{2}\left(v_{0}(x)+\frac{1}{i \omega_{n}\left(\theta_{0}\right)} v_{1}(x)\right) & \text { in } \mathbb{R}^{N},\end{cases}
$$


with the homogenized dispersion tensor $A^{*}$ defined by (1.6) and $\gamma^{+}$defined by (5.1). Similarly, the solution of (1.1) can be written as

$$
u_{\varepsilon}(t, x)=\mathrm{e}^{-i \frac{\omega_{n}\left(\theta_{0}\right) t}{\varepsilon^{2}}} \mathrm{e}^{2 i \pi \frac{\theta_{0} \cdot x}{\varepsilon}} v_{\varepsilon}^{-}(t, x),
$$

where $v_{\varepsilon}^{-}$two-scale converges with drift $-\mathcal{V}$ to $\psi_{n}\left(y, \theta_{0}\right) v^{-}(t, x)$ and $v^{-} \in C([0, T]$; $\left.L^{2}\left(\mathbb{R}^{N}\right)\right)$ is the solution of the homogenized problem

$$
\begin{cases}-2 i \frac{\partial v^{-}}{\partial t}-\operatorname{div}\left(A^{*} \nabla v^{-}\right)+\frac{\gamma^{-}}{\omega_{n}\left(\theta_{0}\right)} v^{-}=0 & \text { in } \mathbb{R}^{N} \times(0, T), \\ v^{-}(t=0, x)=\frac{1}{2}\left(v_{0}(x)-\frac{1}{i \omega_{n}\left(\theta_{0}\right)} v_{1}(x)\right) & \text { in } \mathbb{R}^{N}\end{cases}
$$

Theorem 5.1 gives two different possible limits for the solution $u_{\varepsilon}$. There is no contradiction since these limits corresponds to different convergences and furthermore only one half of the initial data $v_{0}$ is taken into account in each homogenized problem. Each of these asymptotic limits correspond to one of two wave propagating in opposite directions. In other words, Theorem 5.1 is a "weak" justification of (1.8) in the sense that

$$
\begin{aligned}
u_{\varepsilon}(t, x)= & \mathrm{e}^{2 i \pi \frac{\theta_{0} \cdot x}{\varepsilon}} \psi_{n}\left(\frac{x}{\varepsilon}, \theta_{0}\right)\left(\mathrm{e}^{i \frac{\omega_{n}\left(\theta_{0}\right) t}{\varepsilon^{2}}} v^{+}\left(t, x+\frac{\mathcal{V}}{\varepsilon} t\right)+\mathrm{e}^{-i \frac{\omega_{n}\left(\theta_{0}\right) t}{\varepsilon^{2}}} v^{-}\left(t, x-\frac{\mathcal{V}}{\varepsilon} t\right)\right) \\
& +r_{\varepsilon}(t, x),
\end{aligned}
$$

where the remainder term $r_{\varepsilon}$ two-scale converges with drift $-\mathcal{V}$ and $+\mathcal{V}$ to zero. In our companion paper [5], by constructing appropriate correctors in an asymptotic expansion (valid only for smooth coefficients and data), we prove that the remainder term $r_{\varepsilon}$ goes to zero strongly in $L^{2}\left(\mathbb{R}^{N} \times(0, T)\right.$ ), i.e., the above two waves (or limits $v^{+}$and $v^{-}$) completely describe the asymptotic behavior of $u_{\varepsilon}$. We also explain below in Remark 5.5 how the strong convergence of $r_{\varepsilon}$ could be obtained by the method of two-scale convergence (combined with asymptotic expansions).

Remark 5.2 The same result hold true for a system of wave equations (for example, elastodynamics). We never use the fact that we consider a scalar-valued unknown. In particular we do not rely on the maximum principle. Let us simply remark that, even if the original problem is a system of equations, under the simplicity assumption (2.1) for the Bloch eigenvalue, the homogenized problem is always a scalar equations (see [4] for details). If the Bloch eigenvalue is of multiplicity $p$, we expect the homogenized problem to be a system of $p$ equations (see [6] for details).

Proof of Theorem 5.1 The proof is similar in spirit to that of the main result in [6]. We just prove the convergence of $v_{\varepsilon}^{+}$: the case of $v_{\varepsilon}^{-}$is completely symmetric by changing $\omega_{n}\left(\theta_{0}\right)$ in $-\omega_{n}\left(\theta_{0}\right)$, and thus $\mathcal{V}$ in $-\mathcal{V}$. For simplicity we drop the index + and we introduce a new unknown

$$
v_{\varepsilon}(t, x):=\mathrm{e}^{-2 i \pi \frac{\theta_{0} \cdot x}{\varepsilon}} \mathrm{e}^{-i \frac{\omega_{n}\left(\theta_{0}\right) t}{\varepsilon^{2}}} u_{\varepsilon}(t, x) .
$$

By the a priori estimates of Proposition 4.1 it follows that $v_{\varepsilon}$ satisfies the same uniform bounds

$$
\left\|v_{\varepsilon}\right\|_{L^{\infty}\left((0, T) ; L^{2}\left(\mathbb{R}^{N}\right)\right)}+\varepsilon\left\|\nabla v_{\varepsilon}\right\|_{L^{2}\left((0, T) \times \mathbb{R}^{N}\right)^{N}}+\varepsilon^{2}\left\|\frac{\partial v_{\varepsilon}}{\partial t}\right\|_{L^{\infty}\left((0, T) ; L^{2}\left(\mathbb{R}^{N}\right)\right)} \leq C .
$$

Applying the compactness of two-scale convergence with drift (see Proposition 2.4), up to a subsequence, there exists $v^{*}(t, x, y) \in L^{2}\left((0, T) \times \mathbb{R}^{N} ; H^{1}\left(\mathbb{T}^{N}\right)\right)$ such that $v_{\varepsilon}$ and 
$\varepsilon \nabla v_{\varepsilon}$ two-scale converge with drift $\mathcal{V}$ to $v^{*}$ and $\nabla_{y} v^{*}$ respectively. Similarly, by definition of the initial data, $v_{\varepsilon}(0, x)$ two-scale converges (in the usual sense of Proposition 2.3) to $\psi_{n}\left(y, \theta_{0}\right) v_{0}(x)$. The equation satisfied by $v_{\varepsilon}$ is

$$
\begin{cases}\varepsilon^{2} \frac{\partial}{\partial t}\left(\rho_{\varepsilon} \frac{\partial v_{\varepsilon}}{\partial t}\right)+i \sqrt{\lambda_{n}\left(\theta_{0}\right)}\left(\rho_{\varepsilon} \frac{\partial v_{\varepsilon}}{\partial t}+\frac{\partial\left(\rho_{\varepsilon} v_{\varepsilon}\right)}{\partial t}\right)-\frac{\lambda_{n}\left(\theta_{0}\right)}{\varepsilon^{2}} \rho_{\varepsilon} v_{\varepsilon} & \\ -\left(\operatorname{div}+\frac{2 i \pi \theta_{0}}{\varepsilon}\right)\left(A_{\varepsilon}\left(\nabla+\frac{2 i \pi \theta_{0}}{\varepsilon}\right) v_{\varepsilon}\right)=0 & \text { in } \mathbb{R}^{N} \times(0, T), \\ v_{\varepsilon}(t=0, x)=u_{\varepsilon}^{0}(x) \mathrm{e}^{-2 i \pi \frac{\theta_{0} \cdot x}{\varepsilon}} & \text { in } \mathbb{R}^{N}, \\ \frac{\partial v_{\varepsilon}}{\partial t}(t=0, x)=\left(u_{\varepsilon}^{1}(x)-i \frac{\omega_{n}\left(\theta_{0}\right)}{\varepsilon^{2}} u_{\varepsilon}^{0}(x)\right) \mathrm{e}^{-2 i \pi \frac{\theta_{0} \cdot x}{\varepsilon}} & \text { in } \mathbb{R}^{N} .\end{cases}
$$

First step. We multiply (5.8) by the complex conjugate of

$$
\varepsilon^{2} \Phi^{\varepsilon}(t, x)
$$

where

$$
\Phi^{\varepsilon}(t, x):=\Phi\left(t, x+\frac{\mathcal{V}}{\varepsilon} t, \frac{x}{\varepsilon}\right)
$$

and $\Phi(t, x, y)$ is a smooth function defined on $[0, T) \times \mathbb{R}^{N} \times \mathbb{T}^{N}$, with compact support in $[0, T) \times \mathbb{R}^{N}$ and with values in $\mathbb{C}$. In what follows we will denote by $\left(\nabla_{x} \Phi\right)^{\varepsilon}$ the gradient of $\Phi$ with respect to the $x$ variable, evaluated at the point $\left(t, x+\frac{\mathcal{V}}{\varepsilon} t, \frac{x}{\varepsilon}\right)$,

$$
\left(\nabla_{x} \Phi\right)^{\varepsilon}:=\nabla_{x} \Phi\left(t, x+\frac{\mathcal{V}}{\varepsilon} t, \frac{x}{\varepsilon}\right) \text {. }
$$

Integrating by parts we obtain

$$
\begin{aligned}
& -\varepsilon^{2} \int_{\mathbb{R}^{N}} \rho_{\varepsilon}\left(\varepsilon^{2} u_{\varepsilon}^{1}+i \sqrt{\lambda_{n}\left(\theta_{0}\right)} u_{\varepsilon}^{0}\right) \mathrm{e}^{-2 i \pi \frac{\theta_{0} \cdot x}{\varepsilon}} \bar{\Phi}^{\varepsilon}(t=0) \mathrm{d} x \\
& -\varepsilon^{4} \int_{0}^{T} \int_{\mathbb{R}^{N}} \rho_{\varepsilon} \frac{\partial v_{\varepsilon}}{\partial t}\left(\frac{\partial \bar{\Phi}^{\varepsilon}}{\partial t}+\frac{\mathcal{V}}{\varepsilon} \cdot\left(\nabla_{x} \bar{\Phi}\right)^{\varepsilon}\right) \mathrm{d} t \mathrm{~d} x \\
& -2 i \varepsilon^{2} \sqrt{\lambda_{n}\left(\theta_{0}\right)} \int_{0}^{T} \int_{\mathbb{R}^{N}} \rho_{\varepsilon} v_{\varepsilon}\left(\frac{\partial \bar{\Phi}^{\varepsilon}}{\partial t}+\frac{\mathcal{V}}{\varepsilon} \cdot\left(\nabla_{x} \bar{\Phi}\right)^{\varepsilon}\right) \mathrm{d} t \mathrm{~d} x \\
& -i \varepsilon^{4} \sqrt{\lambda_{n}\left(\theta_{0}\right)} \int_{0}^{T} \int_{\mathbb{R}^{N}} \frac{\partial \rho_{1, \varepsilon}}{\partial t} v_{\varepsilon} \bar{\Phi}^{\varepsilon} \mathrm{d} t \mathrm{~d} x \\
& +\int_{0}^{T} \int_{\mathbb{R}^{N}}^{T} A_{\varepsilon}\left(\varepsilon \nabla+2 i \pi \theta_{0}\right) v_{\varepsilon} \cdot\left(\varepsilon \nabla-2 i \pi \theta_{0}\right) \bar{\Phi}^{\varepsilon} \mathrm{d} t \mathrm{~d} x \\
& -\lambda_{n}\left(\theta_{0}\right) \int_{0}^{T} \int_{\mathbb{R}^{N}} \rho_{\varepsilon} v_{\varepsilon} \bar{\Phi}^{\varepsilon} \mathrm{d} t \mathrm{~d} x=0 .
\end{aligned}
$$

Passing to the two-scale limit yields the variational formulation of

$$
-\left(\operatorname{div}_{y}+2 i \pi \theta_{0}\right)\left(A_{0}(y)\left(\nabla_{y}+2 i \pi \theta_{0}\right) v^{*}\right)=\lambda_{n}\left(\theta_{0}\right) \rho_{0}(y) v^{*} \text { in } \mathbb{T}^{N} .
$$


By the simplicity of $\lambda_{n}\left(\theta_{0}\right)$, this implies that there exists a complex-valued function $v(t, x) \in$ $L^{2}\left((0, T) \times \mathbb{R}^{N}\right)$ such that

$$
v^{*}(t, x, y)=v(t, x) \psi_{n}\left(y, \theta_{0}\right) .
$$

Second step. We multiply (5.8) by the complex conjugate of

$$
\Psi_{\varepsilon}(t, x)=\left(\psi_{n}\left(\frac{x}{\varepsilon}, \theta_{0}\right) \phi^{\varepsilon}(t, x)+\frac{\varepsilon}{2 i \pi} \sum_{k=1}^{N} \frac{\partial \psi_{n}}{\partial \theta_{k}}\left(\frac{x}{\varepsilon}, \theta_{0}\right) \frac{\partial \phi^{\varepsilon}}{\partial x_{k}}(t, x)\right)
$$

where

$$
\phi^{\varepsilon}(t, x):=\phi\left(t, x+\frac{\mathcal{V}}{\varepsilon} t\right)
$$

and $\phi(t, x)$ is a smooth, compactly supported, test function defined from $[0, T) \times \mathbb{R}^{N}$ into $\mathbb{C}$. Remark that $\phi^{\varepsilon}$ is not oscillating but is just transported by a large drift. In the sequel we will use the following notations

$$
\begin{aligned}
& \left(\frac{\partial \phi}{\partial t}\right)^{\varepsilon}(t, x):=\frac{\partial \phi}{\partial t}\left(t, x+\frac{\mathcal{V}}{\varepsilon} t\right), \quad\left(\frac{\partial^{2} \phi}{\partial t \partial x_{k}}\right) y^{\varepsilon}(t, x):=\frac{\partial^{2} \phi}{\partial t \partial x_{k}}\left(t, x+\frac{\mathcal{V}}{\varepsilon} t\right) \\
& A_{0, \varepsilon}(x):=A_{0}\left(\frac{x}{\varepsilon}\right) y, \quad A_{1, \varepsilon}(x):=A_{1}\left(t, \frac{t}{\varepsilon}, x, \frac{x}{\varepsilon}\right), \\
& \rho_{0, \varepsilon}(x):=\rho_{0}\left(\frac{x}{\varepsilon}\right), \quad \rho_{1, \varepsilon}(x):=\rho_{1}\left(t, \frac{t}{\varepsilon}, x, \frac{x}{\varepsilon}\right), \\
& \psi_{n}^{\varepsilon}(x):=\psi_{n}\left(\frac{x}{\varepsilon}, \theta_{0}\right) y, \quad \frac{\partial \psi_{n}^{\varepsilon}}{\partial \theta_{k}}=: \frac{\partial \psi_{n}}{\partial \theta_{k}}\left(\frac{x}{\varepsilon}, \theta_{0}\right), \quad \frac{\partial \psi_{n}^{\varepsilon}}{\partial x_{k}}=: \frac{\partial \psi_{n}}{\partial x_{k}}\left(\frac{x}{\varepsilon}, \theta_{0}\right) .
\end{aligned}
$$

Remark that we have $\nabla \phi^{\varepsilon}=(\nabla \phi)^{\varepsilon}$. An integration by parts (with respect to the time variable) yields

$$
\begin{aligned}
& \int_{0}^{T} \int_{\mathbb{R}^{N}}\left(\varepsilon^{2} \frac{\partial}{\partial t}\left(\rho_{\varepsilon} \frac{\partial v_{\varepsilon}}{\partial t}\right)+i \sqrt{\lambda_{n}\left(\theta_{0}\right)}\left(\rho_{\varepsilon} \frac{\partial v_{\varepsilon}}{\partial t}+\frac{\partial\left(\rho_{\varepsilon} v_{\varepsilon}\right)}{\partial t}\right)\right) \bar{\Psi}_{\varepsilon} \mathrm{d} t \mathrm{~d} x \\
& =-\int_{\mathbb{R}^{N}} \rho_{\varepsilon}\left(\varepsilon^{2} u_{\varepsilon}^{1}+i \sqrt{\lambda_{n}\left(\theta_{0}\right)} u_{\varepsilon}^{0}\right) e^{-2 i \pi \frac{\theta_{0} \cdot x}{\varepsilon}} \bar{\Psi}_{\varepsilon}(t=0) \mathrm{d} x \\
& -\int_{0}^{T} \int_{\mathbb{R}^{N}} \rho_{\varepsilon}\left(\varepsilon^{2} \frac{\partial v_{\varepsilon}}{\partial t}+2 i \sqrt{\lambda_{n}\left(\theta_{0}\right)} v_{\varepsilon}\right)\left[\bar{\psi}_{n}^{\varepsilon}\left(\frac{\partial \bar{\phi}}{\partial t}\right)^{\varepsilon}+\frac{\varepsilon}{2 i \pi} \frac{\partial \bar{\psi}_{n}^{\varepsilon}}{\partial \theta_{k}}\left(\frac{\partial^{2} \bar{\phi}}{\partial t \partial x_{k}}\right)^{\varepsilon}\right] \mathrm{d} t \mathrm{~d} x \\
& -\varepsilon \int_{0}^{T} \int_{\mathbb{R}^{N}} \rho_{\varepsilon} \frac{\partial v_{\varepsilon}}{\partial t} \mathcal{V} \cdot \nabla \bar{\phi}^{\varepsilon} \bar{\psi}_{n}^{\varepsilon} \mathrm{d} x \mathrm{~d} t-\frac{2}{\varepsilon} i \sqrt{\lambda_{n}\left(\theta_{0}\right)} \int_{0}^{T} \int_{\mathbb{R}^{N}} \rho_{\varepsilon} v_{\varepsilon} \bar{\psi}_{n}^{\varepsilon} \mathcal{V} \cdot \nabla \bar{\phi}^{\varepsilon} \mathrm{d} x \mathrm{~d} t \\
& +\frac{\varepsilon^{2}}{2 i \pi} \int_{0}^{T} \int_{\mathbb{R}^{N}} \rho_{\varepsilon} \frac{\partial v_{\varepsilon}}{\partial t} \frac{\partial \bar{\psi}_{n}^{\varepsilon}}{\partial \theta_{k}} \mathcal{V} \cdot \nabla \frac{\partial \bar{\phi}^{\varepsilon}}{\partial x_{k}} \mathrm{~d} x \mathrm{~d} t+\frac{1}{\pi} \sqrt{\lambda_{n}\left(\theta_{0}\right)} \int_{0}^{T} \int_{\mathbb{R}^{N}} \rho_{\varepsilon} v_{\varepsilon} \frac{\partial \bar{\psi}_{n}^{\varepsilon}}{\partial \theta_{k}} \mathcal{V} \cdot \nabla \frac{\partial \bar{\phi}^{\varepsilon}}{\partial x_{k}} \mathrm{~d} x \mathrm{~d} t \\
& -\varepsilon^{2} i \sqrt{\lambda_{n}\left(\theta_{0}\right)} \int_{0}^{T} \int_{\mathbb{R}^{N}} \frac{\partial \rho_{1, \varepsilon}}{\partial t} v_{\varepsilon} \bar{\Psi}_{\varepsilon} \mathrm{d} x \mathrm{~d} t .
\end{aligned}
$$


As far as the elliptic part of equation (5.8) is concerned, some algebraic computations similar to those in [6] lead to

$$
\begin{aligned}
\int_{\mathbb{R}^{N}} & {\left[A_{\varepsilon}\left(\nabla+2 i \pi \frac{\theta_{0}}{\varepsilon}\right) v_{\varepsilon} \cdot\left(\nabla-2 i \pi \frac{\theta_{0}}{\varepsilon}\right) \bar{\Psi}_{\varepsilon}-\frac{\lambda_{n}\left(\theta_{0}\right)}{\varepsilon^{2}} \rho_{\varepsilon} v_{\varepsilon} \bar{\Psi}_{\varepsilon}\right] \mathrm{d} x } \\
= & \int_{\mathbb{R}^{N}} A_{0, \varepsilon}\left(\nabla+2 i \pi \frac{\theta_{0}}{\varepsilon}\right)\left(\bar{\phi}^{\varepsilon} v_{\varepsilon}\right) \cdot\left(\nabla-2 i \pi \frac{\theta_{0}}{\varepsilon}\right) y \bar{\psi}_{n}^{\varepsilon} \mathrm{d} x \\
& -\frac{\varepsilon}{2 i \pi} \int_{\mathbb{R}^{N}} A_{0, \varepsilon}\left(\nabla+2 i \pi \frac{\theta_{0}}{\varepsilon}\right)\left(\frac{\partial \bar{\phi}^{\varepsilon}}{\partial x_{k}} v_{\varepsilon}\right) \cdot\left(\nabla-2 i \pi \frac{\theta_{0}}{\varepsilon}\right) \frac{\partial \bar{\psi}_{n}^{\varepsilon}}{\partial \theta_{k}} \mathrm{~d} x \\
& -\int_{\mathbb{R}^{N}} A_{0, \varepsilon} e_{k} \frac{\partial \bar{\phi}^{\varepsilon}}{\partial x_{k}} v_{\varepsilon} \cdot\left(\nabla-2 i \pi \frac{\theta_{0}}{\varepsilon}\right) \bar{\psi}_{n}^{\varepsilon} \mathrm{d} x \\
& +\int_{\mathbb{R}^{N}} A_{0, \varepsilon}\left(\nabla+2 i \pi \frac{\theta_{0}}{\varepsilon}\right)\left(\frac{\partial \bar{\phi}^{\varepsilon}}{\partial x_{k}} v_{\varepsilon}\right) \cdot e_{k} \bar{\psi}_{n}^{\varepsilon} \mathrm{d} x-\int_{\mathbb{R}^{N}} A_{0, \varepsilon} v_{\varepsilon} \nabla \frac{\partial \bar{\phi}^{\varepsilon}}{\partial x_{k}} \cdot e_{k} \bar{\psi}_{n}^{\varepsilon} \mathrm{d} x \\
& +\frac{1}{2 i \pi} \int_{\mathbb{R}^{N}} A_{0, \varepsilon} v_{\varepsilon} \nabla \frac{\partial \bar{\phi}^{\varepsilon}}{\partial x_{k}} \cdot\left(\varepsilon \nabla-2 i \pi \theta_{0}\right) \frac{\partial \bar{\psi}_{n}^{\varepsilon}}{\partial \theta_{k}} \mathrm{~d} x \\
& -\frac{1}{2 i \pi} \int_{\mathbb{R}^{N}} A_{0, \varepsilon} \frac{\partial \bar{\psi}_{n}^{\varepsilon}}{\partial \theta_{k}}\left(\varepsilon \nabla+2 i \pi \theta_{0}\right) v_{\varepsilon} \cdot \nabla \frac{\partial \bar{\phi}^{\varepsilon}}{\partial x_{k}} \mathrm{~d} x-\frac{\lambda_{n}\left(\theta_{0}\right)}{\varepsilon^{2}} \int_{\mathbb{R}^{N}} \rho_{0, \varepsilon} v_{0}^{\varepsilon} \bar{\psi}_{n}^{\varepsilon} \bar{\phi}^{\varepsilon} \mathrm{d} x \\
& \left.+\frac{1}{2 i \pi} \frac{\lambda_{n}\left(\theta_{0}\right)}{\varepsilon} \int_{\mathbb{R}^{N}}\left[\rho_{\mathbb{R}^{N}} \rho_{0, \varepsilon}{v_{1}}_{\varepsilon} \frac{\partial \bar{\phi}^{\varepsilon}}{\partial x_{k}} \frac{\partial \bar{\psi}_{n}^{\varepsilon}}{\partial \theta_{k}} \mathrm{~d} x+2 i \pi \frac{\theta_{0}}{\varepsilon}\right) v_{\varepsilon} \cdot\left(\nabla-2 i \pi \frac{\theta_{0}}{\varepsilon}\right) \bar{\Psi}_{\varepsilon}-\lambda_{n}\left(\theta_{0}\right) \rho_{1, \varepsilon} v_{\varepsilon} \bar{\Psi}_{\varepsilon}\right] \mathrm{d} x
\end{aligned}
$$

Now, for any smooth compactly supported test function $\Phi$ from $\mathbb{R}^{N}$ into $\mathbb{C}$, we deduce from Eq. (1.4) for $\psi_{n}$ that

$$
\int_{\mathbb{R}^{N}} A_{0, \varepsilon}\left(\nabla-2 i \pi \frac{\theta_{0}}{\varepsilon}\right) \bar{\psi}_{n}^{\varepsilon} \cdot\left(\nabla+2 i \pi \frac{\theta_{0}}{\varepsilon}\right) \Phi \mathrm{d} x-\frac{1}{\varepsilon^{2}} \lambda_{n}\left(\theta_{0}\right) \int_{\mathbb{R}^{N}} \rho_{0, \varepsilon} \bar{\psi}_{n}^{\varepsilon} \cdot \Phi \mathrm{d} x=0,
$$

while Eq. (2.5) for $\frac{\partial \psi_{n}}{\partial \theta_{k}}$ implies

$$
\begin{aligned}
& -\frac{\varepsilon}{2 i \pi} \int_{\mathbb{R}^{N}} A_{0, \varepsilon}\left(\nabla-2 i \pi \frac{\theta_{0}}{\varepsilon}\right) \frac{\partial \bar{\psi}_{n}^{\varepsilon}}{\partial \theta_{k}} \cdot\left(\nabla+2 i \pi \frac{\theta_{0}}{\varepsilon}\right) \Phi \mathrm{d} x+\frac{1}{2 i \pi \varepsilon} \lambda_{n}\left(\theta_{0}\right) \int_{\mathbb{R}^{N}} \rho_{0, \varepsilon} \frac{\partial \bar{\psi}_{n}^{\varepsilon}}{\partial \theta_{k}} \Phi \mathrm{d} x \\
& -\int_{\mathbb{R}^{N}} A_{0, \varepsilon}\left(\nabla-2 i \pi \frac{\theta_{0}}{\varepsilon}\right) \bar{\psi}_{n}^{\varepsilon} \cdot e_{k} \Phi \mathrm{d} x+\int_{\mathbb{R}^{N}} A_{0, \varepsilon} e_{k} \bar{\psi}_{n}^{\varepsilon} \cdot\left(\nabla+2 i \pi \frac{\theta_{0}}{\varepsilon}\right) \Phi \mathrm{d} x \\
& +\frac{1}{2 i \pi \varepsilon} \frac{\partial \lambda_{n}}{\partial \theta_{k}}\left(\theta_{0}\right) \int_{\mathbb{R}^{N}} \rho_{0, \varepsilon} \bar{\psi}_{n}^{\varepsilon} \Phi \mathrm{d} x=0 .
\end{aligned}
$$


Equations (5.13) and (5.14) yield some (most welcome!) cancellations in (5.12). The sum of the first and eighth lines of the right hand side of (5.12) cancels out because of (5.13) with $\Phi=\bar{\phi}^{\varepsilon} v_{\varepsilon}$. The second, third, fourth and penultimate lines of the right hand side of (5.12), after integration in time and addition to the fourth line of the right hand side of (5.11), cancel out because of (5.14) with $\Phi=\frac{\partial \bar{\phi}^{\varepsilon}}{\partial x_{k}} v_{\varepsilon}$. On the other hand, we do not change the remaining four terms of (5.12) since they are bounded. Finally, after all these simplifications, (5.8) multiplied by $\bar{\Psi}_{\varepsilon}$ yields

$$
\begin{aligned}
& -\int_{\mathbb{R}^{N}} \rho_{\varepsilon}\left(\varepsilon^{2} u_{\varepsilon}^{1}+i \sqrt{\lambda_{n}\left(\theta_{0}\right)} u_{\varepsilon}^{0}\right) \mathrm{e}^{-2 i \pi \frac{\theta_{0} \cdot x}{\varepsilon}} \bar{\Psi}_{\varepsilon}(t=0) \mathrm{d} x \\
& -\int_{0}^{T} \int_{\mathbb{R}^{N}} \rho_{\varepsilon}\left(\varepsilon^{2} \frac{\partial v_{\varepsilon}}{\partial t}+2 i \sqrt{\lambda_{n}\left(\theta_{0}\right)} v_{\varepsilon}\right) y\left[\bar{\psi}_{n}^{\varepsilon}\left(\frac{\partial \bar{\phi}}{\partial t}\right)^{\varepsilon}+\frac{\varepsilon}{2 i \pi} \frac{\partial \bar{\psi}_{n}^{\varepsilon}}{\partial \theta_{k}}\left(\frac{\partial^{2} \bar{\phi}}{\partial t \partial x_{k}}\right)^{\varepsilon}\right] \mathrm{d} t \mathrm{~d} x \\
& -\varepsilon \int_{0}^{T} \int_{\mathbb{R}^{N}} \rho_{\varepsilon} \frac{\partial v_{\varepsilon}}{\partial t} \mathcal{V} \cdot \nabla \bar{\phi}^{\varepsilon} \bar{\psi}_{n}^{\varepsilon} \mathrm{d} x \mathrm{~d} t+\frac{\varepsilon^{2}}{2 i \pi} \int_{0}^{T} \int_{\mathbb{R}^{N}} \rho_{\varepsilon} \frac{\partial v_{\varepsilon}}{\partial t} \frac{\partial \bar{\psi}_{n}^{\varepsilon}}{\partial \theta_{k}} \mathcal{V} \cdot \nabla \frac{\partial \bar{\phi}^{\varepsilon}}{\partial x_{k}} \mathrm{~d} x \mathrm{~d} t \\
& +\frac{1}{\pi} \sqrt{\lambda_{n}\left(\theta_{0}\right)} \int_{0}^{T} \int_{\mathbb{R}^{N}} \rho_{\varepsilon} v_{\varepsilon} \frac{\partial \bar{\psi}_{n}^{\varepsilon}}{\partial \theta_{k}} \mathcal{V} \cdot \nabla \frac{\partial \bar{\phi}^{\varepsilon}}{\partial x_{k}} \mathrm{~d} x \mathrm{~d} t-\int_{0}^{T} \int_{\mathbb{R}^{N}} A_{0, \varepsilon} v_{\varepsilon} \nabla \frac{\partial \bar{\phi}^{\varepsilon}}{\partial x_{k}} \cdot e_{k} \bar{\psi}_{n}^{\varepsilon} \mathrm{d} x \mathrm{~d} t \\
& +\frac{1}{2 i \pi} \int_{0}^{T} \int_{\mathbb{R}^{N}} A_{0, \varepsilon} v_{\varepsilon} \nabla \frac{\partial \bar{\phi}^{\varepsilon}}{\partial x_{k}} \cdot\left(\varepsilon \nabla-2 i \pi \theta_{0}\right) \frac{\partial \bar{\psi}_{n}^{\varepsilon}}{\partial \theta_{k}} \mathrm{~d} x \mathrm{~d} t \\
& -\frac{1}{2 i \pi} \int_{0}^{T} \int_{\mathbb{R}^{N}} A_{0, \varepsilon} \frac{\partial \bar{\psi}_{n}^{\varepsilon}}{\partial \theta_{k}}\left(\varepsilon \nabla+2 i \pi \theta_{0}\right) v_{\varepsilon} \cdot \nabla \frac{\partial \bar{\phi}^{\varepsilon}}{\partial x_{k}} \mathrm{~d} x \mathrm{~d} t \\
& +\int_{0}^{T} \int_{\mathbb{R}^{N}}\left[A_{1, \varepsilon}\left(\varepsilon \nabla+2 i \pi \theta_{0}\right) v_{\varepsilon} \cdot\left(\varepsilon \nabla-2 i \pi \theta_{0}\right) \bar{\Psi}_{\varepsilon}-\lambda_{n}\left(\theta_{0}\right) \rho_{1, \varepsilon} v_{\varepsilon} \bar{\Psi}_{\varepsilon}\right] \mathrm{d} x \mathrm{~d} t \\
& -\varepsilon^{2} i \sqrt{\lambda_{n}\left(\theta_{0}\right)} \int_{0}^{T} \int_{\mathbb{R}^{N}} \frac{\partial \rho_{1, \varepsilon}}{\partial t} v_{\varepsilon} \bar{\Psi}_{\varepsilon} \mathrm{d} x \mathrm{~d} t=0 .
\end{aligned}
$$

Let us explain how to pass to the limit in (5.15). By assumptions (1.3) on the initial data, the first line of (5.15) satisfies

$$
\begin{aligned}
& \int_{\mathbb{R}^{N}} \rho_{\varepsilon}\left(\varepsilon^{2} u_{\varepsilon}^{1}+i \sqrt{\lambda_{n}\left(\theta_{0}\right)} u_{\varepsilon}^{0}\right) \mathrm{e}^{-2 i \pi \frac{\theta_{0} \cdot x}{\varepsilon}} \bar{\Psi}_{\varepsilon}(t=0) \mathrm{d} x \\
& \rightarrow \int_{\mathbb{R}^{N}} \int_{\mathbb{T}^{N}}\left(i \sqrt{\lambda_{n}\left(\theta_{0}\right)} v_{0}+v_{1}\right) \rho_{0}\left|\psi_{n}\right|^{2} \bar{\phi}(t=0) \mathrm{d} x \mathrm{~d} y .
\end{aligned}
$$


In the second line of (5.15) the only non-zero limit is given by

$$
\int_{0}^{T} \int_{\mathbb{R}^{N}} 2 i \sqrt{\lambda_{n}\left(\theta_{0}\right)} \rho_{\varepsilon} v_{\varepsilon} \bar{\psi}_{n}^{\varepsilon}\left(\frac{\partial \bar{\phi}}{\partial t}\right)^{\varepsilon} \mathrm{d} t \mathrm{~d} x \rightarrow \int_{0}^{T} \int_{\mathbb{R}^{N}} \int_{\mathbb{T}^{N}} 2 i \sqrt{\lambda_{n}\left(\theta_{0}\right)} \rho_{0}\left|\psi_{n}\right|^{2} v \frac{\partial \bar{\phi}}{\partial t} \mathrm{~d} t \mathrm{~d} x \mathrm{~d} y,
$$

while all other terms obviously go to zero. Let us simply indicate that

$$
\int_{0}^{T} \int_{\mathbb{R}^{N}} \varepsilon^{2} \rho_{\varepsilon} \frac{\partial v_{\varepsilon}}{\partial t} \bar{\psi}_{n}^{\varepsilon}\left(\frac{\partial \bar{\phi}}{\partial t}\right)^{\varepsilon} \mathrm{d} t \mathrm{~d} x \rightarrow 0
$$

because $\varepsilon^{2} \frac{\partial v_{\varepsilon}}{\partial t}$, being bounded by virtue of Lemma 4.1, admits a two-scale limit which is necessarily zero since $v_{\varepsilon}$ is bounded in $L^{\infty}\left((0, T) ; L^{2}\left(\mathbb{R}^{N}\right)\right)$.

Let us focus on the third line of (5.15). A second (in time) integration by parts yields

$$
\begin{aligned}
& -\varepsilon \int_{0}^{T} \int_{\mathbb{R}^{N}} \rho_{\varepsilon} \frac{\partial v_{\varepsilon}}{\partial t} \mathcal{V} \cdot \nabla \bar{\phi}^{\varepsilon} \bar{\psi}_{n}^{\varepsilon} \mathrm{d} x \mathrm{~d} t \\
& =\int_{0}^{T} \int_{\mathbb{R}^{N}} \rho_{\varepsilon} v_{\varepsilon}(\mathcal{V} \cdot \nabla)(\mathcal{V} \cdot \nabla) \bar{\phi}^{\varepsilon} \bar{\psi}_{n}^{\varepsilon} \mathrm{d} x \mathrm{~d} t+\varepsilon \int_{0}^{T} \int_{\mathbb{R}^{N}} \rho_{\varepsilon} v_{\varepsilon} \mathcal{V} \cdot \nabla\left(\frac{\partial \bar{\phi}}{\partial t}\right)^{\varepsilon} \bar{\psi}_{n}^{\varepsilon} \mathrm{d} x \mathrm{~d} t \\
& \quad+\varepsilon^{3} \int_{0}^{T} \int_{\mathbb{R}^{N}} \frac{\partial \rho_{1, \varepsilon}}{\partial t} v_{\varepsilon} \mathcal{V} \cdot \nabla \bar{\phi}^{\varepsilon} \bar{\psi}_{n}^{\varepsilon} \mathrm{d} x \mathrm{~d} t+\varepsilon \int_{\mathbb{R}^{N}} \rho_{\varepsilon} v_{\varepsilon}(t=0) \mathcal{V} \cdot \nabla \bar{\phi}(t=0) \bar{\psi}_{n}^{\varepsilon} \mathrm{d} x .
\end{aligned}
$$

On passing to the two-scale limit the last three terms in the right hand side of (5.17) go to zero while the first one gives

$$
-\int_{0}^{T} \int_{\mathbb{R}^{N}} \int_{\mathbb{T}^{N}} \rho_{0}\left|\psi_{n}\right|^{2}(\mathcal{V} \otimes \mathcal{V}) \nabla v \cdot \nabla \bar{\phi} \mathrm{d} x \mathrm{~d} y \mathrm{~d} t
$$

Next, the fourth line in (5.15) tends to zero for the same reason than (5.16). To pass to the limit in the penultimate line of (5.15) we use our assumption on $\rho_{1}, A_{1}$ and Lemma 2.5 which shows that $A_{1}\left(t, \frac{t}{\varepsilon}, x, \frac{x}{\varepsilon}\right)$ and $\rho_{1}\left(t, \frac{t}{\varepsilon}, x, \frac{x}{\varepsilon}\right)$ two-scale converge strongly to $A_{1}^{+}$and $\rho_{1}^{+}$ respectively. By virtue of Proposition 2.4 we can pass to the two-scale limit in the penultimate line of (5.15) under the mere weak two-scale convergence of $v_{\varepsilon}$. The last line of (5.15) goes to zero because $\frac{\partial \rho_{1, \varepsilon}}{\partial t}$ is uniformly bounded by $C \varepsilon^{-1}$. Finally, we can use the weak two-scale convergence with drift $\mathcal{V}$ in all other terms of (5.15) to get

$$
\begin{gathered}
-\int_{\mathbb{R}^{N}} \int_{\mathbb{T}^{N}}\left(i \sqrt{\lambda_{n}\left(\theta_{0}\right)} v_{0}+v_{1}\right) \rho_{0}\left|\psi_{n}\right|^{2} \bar{\phi}(t=0) \mathrm{d} x \mathrm{~d} y \\
-\int_{0}^{T} \int_{\mathbb{R}^{N}} \int_{\mathbb{T}^{N}} 2 i \sqrt{\lambda_{n}\left(\theta_{0}\right)} \rho_{0}\left|\psi_{n}\right|^{2} v \frac{\partial \bar{\phi}}{\partial t} \mathrm{~d} t \mathrm{~d} x \mathrm{~d} y
\end{gathered}
$$




$$
\begin{aligned}
& -\int_{0} \int_{\mathbb{R}^{N}} \int_{\mathbb{T}^{N}} \rho_{0}\left|\psi_{n}\right|^{2}(\mathcal{V} \otimes \mathcal{V}) \nabla v \cdot \nabla \bar{\phi} \mathrm{d} x \mathrm{~d} y \mathrm{~d} t \\
& +\frac{1}{\pi} \sqrt{\lambda_{n}\left(\theta_{0}\right)} \int_{0}^{T} \int_{\mathbb{R}^{N}} \int_{\mathbb{T}^{N}} \rho_{0} \psi_{n} v \frac{\partial \bar{\psi}_{n}}{\partial \theta_{k}} \mathcal{V} \cdot \nabla \frac{\partial \bar{\phi}}{\partial x_{k}} \mathrm{~d} x \mathrm{~d} y \mathrm{~d} t \\
& -\int_{0} \int_{\mathbb{R}^{N}} \int_{\mathbb{T}^{N}} A_{0} \psi_{n} v \nabla \frac{\partial \bar{\phi}}{\partial x_{k}} \cdot e_{k} \bar{\psi}_{n} \mathrm{~d} t \mathrm{~d} x \mathrm{~d} y \\
& +\frac{1}{2 i \pi} \int_{0}^{T} \int_{\mathbb{R}^{N}} \int_{\mathbb{T}^{N}} A_{0} \psi_{n} v \nabla \frac{\partial \bar{\phi}}{\partial x_{k}} \cdot\left(\nabla_{y}-2 i \pi \theta_{0}\right) \frac{\partial \bar{\psi}}{\partial \theta_{k}} \mathrm{~d} t \mathrm{~d} x \mathrm{~d} y \\
& -\frac{1}{2 i \pi} \int_{0}^{T} \int_{\mathbb{R}^{N}} \int_{\mathbb{T}^{N}} A_{0} \frac{\partial \bar{\psi}_{n}}{\partial \theta_{k}}\left(\nabla_{y}+2 i \pi \theta_{0}\right) \psi_{n} v \cdot \nabla \frac{\partial \bar{\phi}}{\partial x_{k}} \mathrm{~d} t \mathrm{~d} x \mathrm{~d} y+\int_{0}^{T} \int_{\mathbb{R}^{N}} \gamma^{+} v \bar{\phi} \mathrm{d} t \mathrm{~d} x \mathrm{~d} y=0 .
\end{aligned}
$$

Recalling formula (2.7) (which is just the compatibility condition or Fredholm alternative for Eq. (2.6), see [4,6] for details) we obtain that the fourth to seventh lines of (5.18) are equal to

$$
\frac{1}{8 \pi^{2}} \int_{0}^{T} \int_{\mathbb{R}^{N}} \nabla_{\theta} \nabla_{\theta} \lambda_{n}\left(\theta_{0}\right) \nabla v \cdot \nabla \bar{\phi} \mathrm{d} t \mathrm{~d} x
$$

Since $\int_{\mathbb{T}^{N}} \rho_{0}\left|\psi_{n}\right|^{2} \mathrm{~d} y=1$ and

$$
A^{*}=\frac{1}{4 \pi^{2}} \nabla_{\theta} \nabla_{\theta} \omega_{n}\left(\theta_{0}\right)=\frac{1}{\sqrt{\lambda_{n}\left(\theta_{0}\right)}}\left(\frac{1}{8 \pi^{2}} \nabla_{\theta} \nabla_{\theta} \lambda_{n}\left(\theta_{0}\right)-\mathcal{V} \otimes \mathcal{V}\right),
$$

(5.18) is therefore equivalent to

$$
\begin{aligned}
& -i \sqrt{\lambda_{n}\left(\theta_{0}\right)} \int_{\mathbb{R}^{N}}\left(v_{0}+\frac{1}{i \sqrt{\lambda_{n}\left(\theta_{0}\right)}} v_{1}\right) \bar{\phi}(t=0) \mathrm{d} x \\
& +\int_{0}^{T} \int_{\mathbb{R}^{N}}\left(-2 i \sqrt{\lambda_{n}\left(\theta_{0}\right)} v \frac{\partial \bar{\phi}}{\partial t}+\sqrt{\lambda_{n}\left(\theta_{0}\right)} A^{*} \nabla v \cdot \nabla \bar{\phi}+\gamma^{+} v \bar{\phi}\right) \mathrm{d} t \mathrm{~d} x=0
\end{aligned}
$$

which is a very weak form of the homogenized equation (5.3). By Lemma 5.4 below, the homogenized problem (5.3) admits a unique solution in $C\left((0, T) ; L^{2}\left(\mathbb{R}^{N}\right)\right)$. The uniqueness of the solution implies that the entire sequence $v_{\varepsilon}$ two-scale converges with drift to $\psi_{n}\left(y, \theta_{0}\right) v(t, x)$.

Remark 5.3 To prove that $v_{\varepsilon}^{+}$two-scale converges with drift $\mathcal{V}$ to $\psi_{n} v^{+}$we only need the hypothesis of "strong average along the group lines" for $\rho_{1}(t, \tau, x, y)$ and $A_{1}(t, \tau, x, y)$ with respect to the sole velocity $+\mathcal{V}$. The symmetric assumption for $-\mathcal{V}$ is required to study the limit of $v_{\varepsilon}^{-}$. If we are interested in constructing approximate travelling wave solutions of (1.1) (instead of solving the Cauchy problem as we did), then we can choose the initial data 
such that $v^{-}(0)=\frac{1}{2}\left(v_{0}-\frac{1}{i \omega_{n}\left(\theta_{0}\right)} v_{1}\right) \equiv 0$, so that only one wave $v^{+}$appears in the ansatz (1.8) and, consequently, only the "strong average along the group lines" assumption for $+\mathcal{V}$ is necessary (see [5] for details).

Lemma 5.4 If the initial data $v_{0}$ belongs to $H^{s}\left(\mathbb{R}^{N}\right)$ (with $s \in \mathbb{N}$ and the usual convention that $H^{0}\left(\mathbb{R}^{N}\right)=L^{2}\left(\mathbb{R}^{N}\right)$ ), the homogenized problem (5.3), resp. (5.5), has a unique solution $v^{+}$, resp. $v^{-}$, in the space $C\left((0, T) ; H^{s}\left(\mathbb{R}^{N}\right)\right)$.

Proof The tensor $A^{*}$ is possibly non-coercive: so, if $v_{0} \in L^{2}\left(\mathbb{R}^{N}\right)$, one can not use the standard elliptic theory to show the existence and uniqueness of a solution in $L^{2}\left((0, T) ; H^{1}\left(\mathbb{R}^{N}\right)\right)$. However, multiplying equation (5.3) by $v^{+}$and taking the imaginary part yields a formal conservation of the $L^{2}\left(\mathbb{R}^{N}\right)$-norm. Therefore, one can use semi-group theory (see e.g. [11] or chapter $\mathrm{X}$ in [27]) to prove existence and uniqueness of a solution $v^{+}$in $C\left((0, T) ; L^{2}\left(\mathbb{R}^{N}\right)\right)$. In general, i.e. if $A^{*}$ is not positive definite, we can not expect any gain in regularity. However, multiplying (5.3) by $(\Delta)^{2 m} v^{+}$, integrating by parts and taking the imaginary part we also get a formal conservation of the norm $\left\|(\Delta)^{m} v^{+}(t)\right\|_{L^{2}\left(\mathbb{R}^{N}\right)}$. A similar argument works for odd powers of $\Delta$. Therefore, $v_{0} \in H^{s}\left(\mathbb{R}^{N}\right)$ implies that $v^{+} \in C\left((0, T) ; H^{s}\left(\mathbb{R}^{N}\right)\right)$. Of course, the same holds true for (5.5).

Remark 5.5 By the method of two-scale convergence it is possible to improve Theorem 5.1 by proving that $r_{\varepsilon}$, defined in (5.6), goes to zero strongly. However, since it would require some technical efforts and since such a result is proved in greater generality in our companion paper [5], we do not give a full proof and merely sketch the main ideas. The key point is to use the notion of strong two-scale convergence (see Theorem 1.8 in [1]) which says that a sequence $v_{\varepsilon} \in L^{2}\left(\mathbb{R}^{N}\right)$, two-scale converging to a limit $v^{*}(x, y)$ and such that

$$
\lim _{\varepsilon \rightarrow 0}\left\|v_{\varepsilon}\right\|_{L^{2}\left(\mathbb{R}^{N}\right)}=\left\|v^{*}\right\|_{L^{2}\left(\mathbb{R}^{N} \times \mathbb{T}^{N}\right)},
$$

satisfies (with a minor technical smoothness assumption on $v^{*}$ )

$$
\lim _{\varepsilon \rightarrow 0}\left\|v_{\varepsilon}(x)-v^{*}\left(x, \frac{x}{\varepsilon}\right)\right\|_{L^{2}\left(\mathbb{R}^{N}\right)}=0 .
$$

We want to apply such a result to $u_{\varepsilon}$ but the required energy convergence is not straightforward since the total energy (4.3) involves only derivatives of $u_{\varepsilon}$. Therefore, we work with the energy conservation of $w_{\varepsilon}$, the time primitive of $u_{\varepsilon}$ introduced in (4.5). The proof of the strong convergence of $r_{\varepsilon}$ is divided in five steps as follows.

Since the initial data of $w_{\varepsilon}$ involves the solution $\chi_{\varepsilon}$ of (4.6), the first step is to show that $\left\|\chi_{\varepsilon}\right\|_{L^{2}\left(\mathbb{R}^{N}\right)} \leq C \varepsilon^{2}$ (see Remark 4.3 for details).

The second step amounts to prove the same estimate for $w_{\varepsilon}$, that is

$$
\left\|w_{\varepsilon}\right\|_{L^{\infty}\left((0, T) ; L^{2}\left(\mathbb{R}^{N}\right)\right)} \leq C \varepsilon^{2} .
$$

This can be achieved by introducing a new time regularization

$$
z_{\varepsilon}(t, x)=\mathrm{e}^{-\gamma t}\left(\int_{0}^{t} \mathrm{e}^{\gamma s} w_{\varepsilon}(s, x) \mathrm{d} s+\zeta_{\varepsilon}(x)\right),
$$

where $\zeta_{\varepsilon}$ is defined as the unique solution in $H^{1}\left(\mathbb{R}^{N}\right)$ of

$$
-\operatorname{div}\left(A_{\varepsilon}(0) \nabla \zeta_{\varepsilon}\right)+\gamma^{2} \varepsilon^{2} \rho_{\varepsilon}(0) \zeta_{\varepsilon}=-\varepsilon^{2} \rho_{\varepsilon}(0) \frac{\partial w_{\varepsilon}}{\partial t}(0)+\gamma \varepsilon^{2} \rho_{\varepsilon}(0) w_{\varepsilon}(0) \text { in } \mathbb{R}^{N} .
$$


It is easily seen that (5.20) is similar to (4.6) except that its right-hand side is multiplied by a factor $\varepsilon^{2}$. Thus, the arguments of Proposition 4.2 and Remark 4.3 yield that

$$
\left\|\zeta_{\varepsilon}\right\|_{L^{2}\left(\mathbb{R}^{N}\right)}+\varepsilon\left\|\nabla \zeta_{\varepsilon}\right\|_{L^{2}\left(\mathbb{R}^{N}\right)^{N}} \leq C \varepsilon^{4}
$$

Then, proceeding as in the proof of Proposition 4.1, we write the energy balance for $z_{\varepsilon}$, which satisfies the same wave equation (4.9) as $w_{\varepsilon}$ with different initial data and source term, smaller of order $\varepsilon^{2}$ than those of (4.9). We thus deduce the following estimate

$$
\left\|\frac{\partial z_{\varepsilon}}{\partial t}\right\|_{L^{\infty}\left((0, T) ; L^{2}\left(\mathbb{R}^{N}\right)\right)} \leq C \varepsilon^{2},
$$

which in turn implies $\left\|z_{\varepsilon}\right\|_{L^{\infty}\left((0, T) ; L^{2}\left(\mathbb{R}^{N}\right)\right)} \leq C \varepsilon^{2}$ because $z_{\varepsilon}(0)=\zeta_{\varepsilon}$. Since, similarly to (4.11), we have $w_{\varepsilon}=\frac{\partial z_{\varepsilon}}{\partial t}+\gamma z_{\varepsilon}$, we deduce the desired estimate (5.19) for $w_{\varepsilon}$.

The third step is to show the equipartition of the energy for $w_{\varepsilon}$, namely

$$
E\left(w_{\varepsilon}(t)\right)=\int_{\mathbb{R}^{N}} \varepsilon^{2} \rho_{\varepsilon}\left|\frac{\partial w_{\varepsilon}}{\partial t}(t, x)\right|^{2} \mathrm{~d} x+\mathcal{O}\left(\varepsilon^{3}\right) .
$$

The fourth step is to combine the energy conservation (4.10) for $w_{\varepsilon}$, the previous energy equipartition and the estimate (5.19) to obtain

$$
\int_{\mathbb{R}^{N}} \rho_{\varepsilon}\left|\frac{\partial w_{\varepsilon}}{\partial t}\right|^{2} \mathrm{~d} x+\mathcal{O}(\varepsilon)=\varepsilon^{-2} E\left(w_{\varepsilon}(0)\right) .
$$

Recalling (4.11), $u_{\varepsilon}=\frac{\partial w_{\varepsilon}}{\partial t}+\gamma w_{\varepsilon}$, using estimate (5.19) for $w_{\varepsilon}$, and passing to the two-scale limit in the right-hand side of (5.21), after some tedious but easy algebra we obtain

$$
\lim _{\varepsilon \rightarrow 0} \int_{\mathbb{R}^{N}} \rho_{\varepsilon}\left|u_{\varepsilon}\right|^{2} \mathrm{~d} x=\left\|v^{+}(0)\right\|_{L^{2}\left(\mathbb{R}^{N}\right)}^{2}+\left\|v^{-}(0)\right\|_{L^{2}\left(\mathbb{R}^{N}\right)}^{2} .
$$

The fifth and last step is to show that $r_{\varepsilon}$, defined by (5.6), converges strongly to zero in $L^{2}\left(\mathbb{R}^{N}\right)$. By its definition (5.6), for a.e. $t \in(0, T)$, we have

$$
\begin{aligned}
\int_{\mathbb{R}^{N}} \rho_{\varepsilon}\left|r_{\varepsilon}\right|^{2} \mathrm{~d} x= & \int_{\mathbb{R}^{N}} \rho_{\varepsilon}\left|u_{\varepsilon}\right|^{2} \mathrm{~d} x-2 \int_{\mathbb{R}^{N}} \rho_{\varepsilon} u_{\varepsilon}\left(u_{\varepsilon}-r_{\varepsilon}\right) \mathrm{d} x \\
& +\int_{\mathbb{R}^{N}} \rho_{\varepsilon}\left|\psi_{n}\left(\frac{x}{\varepsilon}, \theta_{0}\right)\right|^{2}\left(\left|v^{+}\left(t, x+\frac{\mathcal{V}}{\varepsilon} t\right)\right|^{2}+\left|v^{-}\left(t, x-\frac{\mathcal{V}}{\varepsilon} t\right)\right|^{2}\right) \mathrm{d} x \\
& +\int_{\mathbb{R}^{N}} \rho_{\varepsilon}\left|\psi_{n}\left(\frac{x}{\varepsilon}, \theta_{0}\right)\right|^{2} \mathrm{e}^{2 i \frac{\omega_{n}\left(\theta_{0}\right) t}{\varepsilon^{2}}} v^{+}\left(y t, x+\frac{\mathcal{V}}{\varepsilon} t\right) \bar{v}^{-}\left(t, x-\frac{\mathcal{V}}{\varepsilon} t\right) \mathrm{d} x \\
& +\int_{\mathbb{R}^{N}} \rho_{\varepsilon}\left|\psi_{n}\left(\frac{x}{\varepsilon}, \theta_{0}\right)\right|^{2} \mathrm{e}^{-2 i \frac{\omega_{n}\left(\theta_{0}\right) t}{\varepsilon^{2}}} \bar{v}^{+}\left(t, x+\frac{\mathcal{V}}{\varepsilon} t\right) v^{-}\left(t, x-\frac{\mathcal{V}}{\varepsilon} t\right) \mathrm{d} x .
\end{aligned}
$$

In the case when $\mathcal{V} \neq 0$, for $\varepsilon$ sufficiently small, the last two lines of the right-hand side of (5.23) converge to zero because the functions $v^{+}, v^{-}$belong to $L^{2}\left(\mathbb{R}^{N}\right)$ and, if translated away by a large drift, their product converges strongly to zero. The first term in the right-hand 
side of (5.23) converges by (5.22) and we can pass to the two-scale limit (with drift) in the remaining terms of (5.23) to get, for a.e. $t \in(0, T)$,

$$
\lim _{\varepsilon \rightarrow 0} \int_{\mathbb{R}^{N}} \rho_{\varepsilon}\left|r_{\varepsilon}\right|^{2} \mathrm{~d} x=0 .
$$

In the case when $\mathcal{V}=0$, the previous argument of "disjoint supports" in the limit does not work for $v^{+}, v^{-}$. Rather, we observe that in the last two lines of the right-hand side of (5.23) $v^{+}$and $v^{-}$are multiplied by functions oscillating in time. Therefore if we integrate (5.23) with respect to time, again these two terms converge to zero, and we obtain

$$
\lim _{\varepsilon \rightarrow 0} \int_{0}^{T} \int_{\mathbb{R}^{N}} \rho_{\varepsilon}\left|r_{\varepsilon}\right|^{2} \mathrm{~d} x \mathrm{~d} t=0
$$

\section{Link with geometric optics and the WKB method}

We make a comparison with the so-called WKB method (Wentzel, Kramers, Brillouin) which is well known for studying high frequency limits of the wave equation (see e.g. [9]). We content ourselves in recalling well-known formal asymptotic expansions. The WKB method is concerned with a shorter time scale than that of (1.1). More precisely, in this section we rescale the time variable which is now $\tau=\varepsilon^{-1} t$ and we consider the following wave equation

$$
\begin{cases}\rho\left(\frac{x}{\varepsilon}\right) \frac{\partial^{2} v_{\varepsilon}}{\partial \tau^{2}}-\operatorname{div}\left(A\left(\frac{x}{\varepsilon}\right) \nabla v_{\varepsilon}\right)=0 & \text { in } \mathbb{R}^{N} \times \mathbb{R}^{+}, \\ v_{\varepsilon}(0, x)=v_{\varepsilon}^{0}(x) & \text { in } \mathbb{R}^{N}, \\ \frac{\partial v_{\varepsilon}}{\partial \tau}(0, x)=v_{\varepsilon}^{1}(x) & \text { in } \mathbb{R}^{N},\end{cases}
$$

with high frequency initial data

$v_{\varepsilon}^{0}(x)=\varepsilon \mathrm{e}^{2 i \pi \frac{S^{0}(x)}{\varepsilon}} v_{0}(x) \psi_{n}\left(\frac{x}{\varepsilon}, \nabla S^{0}(x)\right) \quad$ and $\quad v_{\varepsilon}^{1}(x)=\mathrm{e}^{2 i \pi \frac{S^{0}(x)}{\varepsilon}} v_{1}(x) \psi_{n}\left(\frac{x}{\varepsilon}, \nabla S^{0}(x)\right)$

where $S^{0}(x)$ is the initial phase and $\psi_{n}(y, \theta)$ is the $n$th eigenfunction of the Bloch spectral problem (1.4). The geometric optic or WKB ansatz is

$$
v_{\varepsilon}(\tau, x)=\varepsilon \mathrm{e}^{2 i \pi \frac{S(\tau, x)}{\varepsilon}}\left(w\left(\tau, x, \frac{x}{\varepsilon}\right)+\varepsilon w_{1}\left(\tau, x, \frac{x}{\varepsilon}\right)+\varepsilon^{2} \ldots\right)
$$

where $w(\tau, x, y)$ and $w_{1}(\tau, x, y)$ are periodic functions with respect to the last variable $y \in \mathbb{T}^{N}$. The ansatz first order derivatives are

$$
\begin{aligned}
\frac{\partial v_{\varepsilon}}{\partial \tau} & =\mathrm{e}^{2 i \pi \frac{S(\tau, x)}{\varepsilon}}\left(2 i \pi\left(w+\varepsilon w_{1}\right) \frac{\partial S}{\partial \tau}+\varepsilon \frac{\partial w}{\partial \tau}+\varepsilon^{2} \ldots\right), \\
\nabla v_{\varepsilon} & =\mathrm{e}^{2 i \pi \frac{S(\tau, x)}{\varepsilon}}\left(2 i \pi\left(w+\varepsilon w_{1}\right) \nabla S+\nabla_{y} w+\varepsilon\left(\nabla_{x} w+\nabla_{y} w_{1}\right)+\varepsilon^{2} \ldots\right) .
\end{aligned}
$$

Plugging this ansatz in the wave equation (6.1) yields, at least formally, a cascade of equations in terms of powers of $\varepsilon$. The first and higher order, $\varepsilon^{-1}$, is

$$
-\left(\operatorname{div}_{y}+2 i \pi \nabla S\right)\left(A(y)\left(\nabla_{y}+2 i \pi \nabla S\right) w\right)=4 \pi^{2} \rho(y)\left(\frac{\partial S}{\partial \tau}\right)^{2} w \quad \text { in } \quad \mathbb{T}^{N} .
$$

For given $(\tau, x),(6.2)$ is precisely the Bloch spectral equation, a p.d.e. with respect to the $y$ variable, with the Bloch frequency $\theta:=\nabla S(\tau, x)$. In view of the initial data and because of 
assumption (2.1) on the simplicity of $\lambda_{n}(\theta)$, we deduce that necessarily the solution of (6.2) is a multiple of the $n$th eigenfunction

$$
w(\tau, x, y)=v(\tau, x) \psi_{n}(y, \nabla S(\tau, x))
$$

with the corresponding eigenvalue

$$
4 \pi^{2}\left(\frac{\partial S}{\partial \tau}\right)^{2}=\lambda_{n}(\nabla S)
$$

It turns out that (6.3) is equivalent to two eikonal or Hamilton-Jacobi equations

$$
2 \pi \frac{\partial S}{\partial \tau}= \pm \sqrt{\lambda_{n}(\nabla S)}
$$

with the initial data $S(0, x)=S^{0}(x)$, which allows us to compute the phase $S(\tau, x)$ at least as far as smooth solutions of (6.3) exist.

The next order, $\varepsilon^{0}$, yields

$$
-\left(\operatorname{div}_{y}+2 i \pi \nabla S\right)\left(A(y)\left(\nabla_{y}+2 i \pi \nabla S\right) w_{1}\right)=\lambda_{n}(\nabla S) \rho(y) w_{1}+f \quad \text { in } \quad \mathbb{T}^{N},
$$

with

$$
f(\tau, x, y)=-4 i \pi \rho(y) \frac{\partial S}{\partial \tau} \frac{\partial w}{\partial \tau}+\left(\operatorname{div}_{y}+2 i \pi \nabla S\right)\left(A \nabla_{x} w\right)+\operatorname{div}_{x}\left(A\left(\nabla_{y}+2 i \pi \nabla S\right) w\right) .
$$

To solve (6.4) for $w_{1}$, the Fredholm alternative requires that

$$
\int_{\mathbb{T}^{N}} \rho(y) f(t, x, y) \bar{\psi}_{n}(y, \nabla S) \mathrm{d} y=0 .
$$

After some computations and using the Fredholm alternative for (2.5), (6.5) is equivalent to the following homogenized transport equation

$$
\frac{\partial v}{\partial \tau} \pm \mathcal{V} \cdot \nabla_{x} v \pm b^{*} v=0
$$

with the group velocity given again by formula (1.5), i.e.

$$
\mathcal{V}=\frac{\nabla_{\theta} \lambda_{n}(\nabla S)}{4 \pi \sqrt{\lambda_{n}(\nabla S)}}=\frac{1}{2 \pi} \nabla_{\theta}\left(\sqrt{\lambda_{n}(\nabla S)}\right)
$$

and $b^{*}(t, x)$ a (complicated) attenuation coefficient. Overall, the WKB method shows that formally

$$
\begin{aligned}
v_{\varepsilon}(\tau, x) \approx & \varepsilon \mathrm{e}^{2 i \pi \frac{S^{+}(\tau, x)}{\varepsilon}} \psi_{n}\left(\frac{x}{\varepsilon}, \nabla S^{+}(\tau, x)\right) v^{+}(\tau, x) \\
& +\varepsilon \mathrm{e}^{2 i \pi \frac{S^{-}(\tau, x)}{\varepsilon}} \psi_{n}\left(\frac{x}{\varepsilon}, \nabla S^{-}(\tau, x)\right) v^{-}(\tau, x)
\end{aligned}
$$

where $S^{ \pm}$are the solutions of the two eikonal equations, $v^{ \pm}$are the solutions of the two transport equations with group velocities $\pm \mathcal{V}$. Actually, the WKB method works for periodically modulated coefficients. It can be rigorously justified only for short times (before caustics). However, the transport equation (6.6) can be rigorously established, globally in time, by the method of Wigner measures [19] which is a generalization of $H$-measures [18,31] to the periodic case. 
A special case, of particular interest for the present work, is obtained for purely periodic coefficients and monochromatic initial data. More precisely, if we assume that $S^{0}(x)=\theta \cdot x$, then the explicit (and globally defined) solutions of the two eikonal equations are

$$
S^{+}(\tau, x)=\theta \cdot x+\sqrt{\lambda_{n}(\theta)} \tau \quad \text { and } \quad S^{-}(\tau, x)=\theta \cdot x-\sqrt{\lambda_{n}(\theta)} \tau .
$$

Furthermore, the group velocity $\mathcal{V}$ is constant and $b^{*}=0$, so that

$$
v^{ \pm}(\tau, x)=v_{0}^{ \pm}(x \pm \mathcal{V} \tau) \quad \text { with } \quad v_{0}^{ \pm}=\frac{1}{2}\left(v_{0} \pm \frac{1}{i \omega_{n}(\theta)} v_{1}\right)
$$

In such a case the approximate formula (6.7) reduces to

$$
v_{\varepsilon}(\tau, x) \approx \varepsilon \mathrm{e}^{2 i \pi \frac{\theta \cdot x}{\varepsilon}} \psi_{n}\left(\frac{x}{\varepsilon}, \theta\right)\left(\mathrm{e}^{i \frac{\sqrt{\lambda_{n}(\theta) \tau}}{\varepsilon}} v_{0}^{+}(x+\mathcal{V} \tau)+\mathrm{e}^{-i \frac{\sqrt{\lambda_{n}(\theta) \tau}}{\varepsilon}} v_{0}^{-}(x-\mathcal{V} \tau)\right)
$$

which can be directly compared to our result in Theorem 3.2. After changing the time scale, i.e. replacing $\tau$ bt $t / \varepsilon$, the main difference between (6.8) and (1.8) is that the envelope functions are fixed, given by the initial data, in (6.8), while they evolve according to Schrödinger equation in (3.4).

Our results of Sect. 5 appears as a generalization and an extension of the WKB method for longer times in the case of monochromatic wave packets. Theorems 3.2 and 5.1 recover the WKB transport effect but they further describe how the envelope function of the wave packet is deformed with time, according to a Schrödinger equation.

Acknowledgments The work of G. Allaire and M. Palombaro was partly supported by the MULTIMAT european network MRTN-CT-2004-505226 funded by the EEC. The research of G. Allaire is also partially supported by the DEFI project at INRIA Saclay Ile de France. The research of J. Rauch is partially supported by the U.S. National Science Foundation under grant NSF-DMS-0104096. M. Palombaro and J. Rauch thank the CMAP at the École Polytechnique and its members for their hospitality.

\section{References}

1. Allaire, G.: Homogenization and two-scale convergence. SIAM J. Math. Anal. 23(6), 1482-1518 (1992)

2. Allaire, G.: Dispersive limits in the homogenization of the wave equation. Annales de la Faculté Des Sciences de Toulouse XII, 415-431 (2003)

3. Allaire, G., Amar, M.: Boundary layer tails in periodic homogenization, ESAIM COCV 4, pp. 209-243 (1999)

4. Allaire, G., Capdeboscq, Y., Piatnistki, A., Siess, V., Vanninathan, M.: Homogenization of periodic systems with large potentials. Archive Rat. Mech. Anal. 174, 179-220 (2004)

5. Allaire, G., Palombaro, M., Rauch, J.: Diffractive geometric optics for Bloch wave packets, preprint

6. Allaire, G., Piatnistki, A.: Homogenization of the Schrödinger equation and effective mass theorems. Comm. Math Phys. 258, 1-22 (2005)

7. Bamberger, A., Engquist, B., Halpern, L., Joly, P.: Parabolic wave equation approximations in heterogenous media. SIAM J. Appl. Math. 48(1), 99-128 (1988)

8. Benisty, H., Weisbuch, C.: Photonic crystals. In: Wolf, E. (ed.) Progress in optics, vol. 49, pp. 177-313. Elsevier, Amsterdam (2006)

9. Bensoussan, A., Lions, J.-L., Papanicolaou, G.: Asymptotic analysis for periodic structures. NorthHolland, Amsterdam (1978)

10. Brahim-Otsmane, S., Francfort, G., Murat, F.: Correctors for the homogenization of the wave and heat equations. J. Math. Pures Appl. 71(9), 197-231 (1992)

11. Brézis, H.: Opérateurs maximaux monotones et semi-groupes de contractions dans les espaces de Hilbert. North-Holland, Amsterdam (1973)

12. Conca, C., Planchard, J., Vanninathan, M.: Fluids and periodic structures, RMA, vol. 38. Wiley \& Masson, Paris (1995)

13. Conca, C., Orive, R., Vanninathan, M.: On Burnett coefficients in strongly periodic media (preprint) 
14. Donnat, P., Joly, J.-L., Metivier, G., Rauch, J.: Diffractive nonlinear geometric optics, Séminaire sur les Equations aux Dérivées Partielles, 1995-1996, Exp. No. XVII, p. 25. Ecole Polytechnique, Palaiseau (1996)

15. Donnat, P., Rauch, J.: Modeling the dispersion of light, Singularities and oscillations (Minneapolis, MN, 1994/1995), IMA Vol. Math. Appl., vol. 91, pp. 17-35. Springer, New York (1997)

16. Donnat, P., Rauch, J.: Dispersive nonlinear geometric optics. J. Math. Phys. 38(3), 1484-1523 (1997)

17. Francfort, G., Murat, F.: Oscillations and energy densities in the wave equation. Comm. Partial Differ. Equ. 17, 1785-1865 (1992)

18. Gérard, P.: Microlocal defect measures. Comm. Partial Differ. Equ. 16, 1761-1794 (1991)

19. Gérard, P., Markowich, P., Mauser, N., Poupaud, F.: Homogenization limits and Wigner transforms. Comm. Pure Appl. Math. 50(4), 323-379 (1997)

20. Kato, T.: Perturbation theory for linear operators. Springer, Berlin (1966)

21. Kuchment, P.: Floquet theory for partial differential equations, Operator Theory: Advances and Applications, vol. 60. Birkhäuser Verlag, Basel (1993)

22. Kuchment, P.: The mathematics of photonic crystals, Mathematical modeling in optical science, Frontiers Appl. Math., vol. 22, pp. 207-272. SIAM, Philadelphia (2001)

23. Leontovich, M., Fock, V.: Solution of the problem of propagation of electromagnetic waves along the earth's surface by the method of parabolic equation. Acad. Sci. USSR. J. Phys. 10, 13-24 (1946)

24. de Martin Sterke, C., Sipe, J.: Envelope-function approach for the electrodynamics of nonlinear periodic structures. Phys. Rev. A 38, 5149-5165 (1998)

25. Marusic-Paloka, E., Piatnitski, A.: Homogenization of nonlinear convection-diffusion equation with rapidly oscillating coefficients and strong convection. J. Lond. Math. Soc. 72(2), 391-409 (2005)

26. Nguetseng, G.: A general convergence result for a functional related to the theory of homogenization. SIAM J. Math. Anal. 20(3), 608-623 (1989)

27. Reed, M., Simon, B.: Methods of Modern Mathematical Physics. Academic Press, New York (1978)

28. Santosa, F., Symes, W.: A dispersive effective medium for wave propagation in periodic composites. SIAM J. Appl. Math. 51, 984-1005 (1991)

29. Sipe, J., Winful, H.: Nonlinear Schrödinger solitons in a periodic structure. Opt. Lett. 13, 132-133 (1988)

30. Tappert, F.: The parabolic approximation method, in Wave propagation and underwater acoustics (Workshop, Mystic, Conn., 1974). Lecture Notes in Physics, vol. 70, pp. 224-287. Springer, Berlin (1977)

31. Tartar, L.: H-measures, a new approach for studying homogenization, oscillations and concentration effects in partial differential equations. Proc. R. Soc. Edinburgh 115A, 193-230 (1990) 